\title{
Role of electromagnetically induced transparency in resonant four-wave-mixing schemes
}

\author{
J. C. Petch, C. H. Keitel, P. L. Knight, and J. P. Marangos \\ Optics Section, Blackett Laboratory, Imperial College, London SW7 2BZ, England
}

(Received 29 June 1995)

\begin{abstract}
The effect of electromagnetically induced transparency in resonant four-wave-mixing schemes is investigated in an analysis that goes beyond perturbation theory in the coherent driving field. In addition we examine the case where the two-photon pump field is sufficiently strong to necessitate a nonperturbative treatment. This allows us to examine the cases where either one or both of the driving fields are strong. Phase matching is included in a plane-wave propagation treatment that matches the situation most likely to be encountered in actual experiments. The calculations are in part intended to model real experimental situations and thus incorporate driving and pump-field linewidths via the phase-diffusion model and Doppler broadening. With a strong pump-field laser, large enhancements in the efficiency of light generation occur at frequencies corresponding to the Autler-Townes satellites induced by the strong driving field. In this situation gain and high four-wave-mixing efficiency are simultaneously present, resulting in the production of a large intensity of coherent radiation.
\end{abstract}

PACS number(s): 42.50.Gy, 42.50.Hz, 42.50.Md

\section{INTRODUCTION}

The observation of electromagnetically induced transparency (EIT) has clearly shown that an atomic medium can be nonabsorptive with respect to a laser field even though the frequency of this field is within the linewidth of an atomic transition of this medium [1]. The origin of this effect may be traced back to population trapping in a decoupled superposition of ground states, or alternatively viewed in terms of destructively interfering absorption pathways due to the nonlinearities of the additional preparation laser field on a different connected transition. It has triggered the discovery of many related phenomena, in particular lasing without inversion [2]. Obviously quantum interference effects can occur in an equivalent way for the stimulated emission process, however the involvement of a third or fourth level in the coherent lasing process (coupled via additional preparation laser fields) can disturb the symmetry between stimulated emission and absorption. Stimulated absorption may therefore be reduced more effectively than the competing stimulated emission at a particular frequency, thus leading to laser action with less population in the upper level than in the ground state; see, e.g., [3] and for the experimental realization [4]. As a further application it was pointed out that preparing a medium with a laser field on one transition may not only affect the absorption and emission processes on a different connected transition but also the dispersive properties. In particular the index of refraction may be maximal at that frequency where the absorption is canceled [5]. Other peculiar properties of media involving EIT include subPoissonian statistics of radiation emitted by such systems [6]; for a more complete treatment of related phenomena we recommend various review articles [7].

The work presented here is concerned with the possibility of enhancing nonlinear optical processes using EIT as predicted first by Harris et al. [8] and investigated experimentally by Hakuta et al. [9,10] and Jain et al. [11]. Harris et al. considered the efficiency of four-wave mixing on one transition of a three-level system while inducing EIT with a strong laser field on a different transition and pumping with a weak coherent field on the remaining two-photon transition. These authors keep the nonlinearities of the strong field responsible for inducing EIT to all orders and show that the reduction of the absorption coefficient can increase the efficiency of fourwave mixing substantially. In the present paper we continue the study of four-wave mixing and its enhancement by EIT, and retain the nonlinearities of the coherent pump field on the two-photon transition to all orders. We show that the efficiency of four-wave mixing can be increased even more drastically, in particular when in addition to strong driving field the two-photon pump field also becomes strong. Related effects for the single-atom response have been reported by other workers [12], but they do not include, as we do, laser linewidth effects or realistic phase matching in their analysis. We also show that if realistic phase matching is included this efficiency is described more generally by a ratio of the nonlinear and linear susceptibilities $\left|\chi^{(3)}\right| /\left|\chi^{(1)}\right|$ valid for arbitrary detunings, which reduces to the parameter proposed earlier in $[8]$ (i.e., $\left|\chi^{(3)}\right| / \operatorname{Im}\left[\chi^{(1)}\right]$ ) when the resonance condition is applied.

Our calculations are aimed in part at modeling specific experimental situations so that attention is given to the estimation of the output of four-wave mixing under the influence of the various unavoidable coherence-degrading processes such as phase fluctuations of the laser field (leading to a finite laser bandwidth) and also in the presence of Doppler broadening (due to the finite temperature of the gas or vapor). We concentrate on the case of resonant four-wave sumdifference mixing schemes. In these the three levels are coupled in a scheme (see Fig. 1) with the pump field exciting a two-photon transition between the ground and upper excited level (separated by $2 \hbar \omega_{p}$ ) and a strong field at frequency $\omega_{d}$ (which we will refer to as the driving field) coupling the two upper levels in the scheme with the possibility of generating a field at the frequency $\omega_{l}$ corresponding to the allowed transition between the lower excited state and the ground state. These schemes are realizable in practice in a number of atomic systems (if hyperfine structure is ig- 


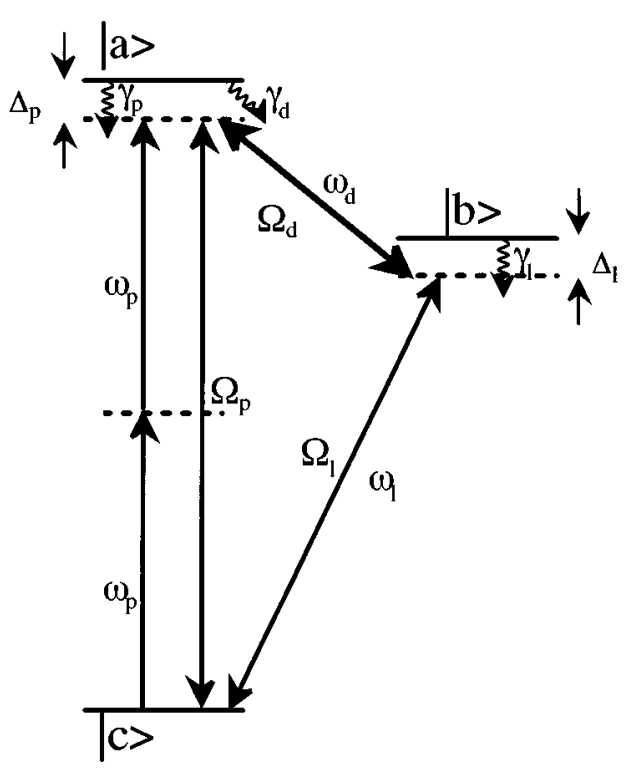

FIG. 1. The three-level scheme under consideration: Four-wave mixing is investigated on the $b-c$ transition while a strong laser field with Rabi frequency $\Omega_{d}$ on the $a-b$ transition may lead to induced transparency and a further coherent pump field with Rabi frequency $\Omega_{p}$ on the $a-c$ transition closes the loop and supplies population in the upper level.

nored). For instance the noble gases (e.g., $\mathrm{Kr}$ and $\mathrm{Xe}$ ) form a three-level system (between the $4 p^{6}$ ground state and excited states of the $5 p 4 p^{5}$ and $5 s 4 p^{5}$ configurations in $\mathrm{Kr}$, for example) for which $\omega_{d}$ is in the near IR, $\omega_{p}$ in the UV, and $\omega_{l}$ in the VUV. It is this fact that makes these schemes interesting as candidates for investigation of the enhancement of coherent VUV generation through the operation of EIT.

The intensity generated in any coherent nonlinear frequency mixing process will scale quadratically with the atomic number density. To optimize the generation efficiency it is therefore usually necessary to work with relatively high density gases or vapors, contained either within a cell or in a gas jet. In these situations, however, we cannot avoid the effects of Doppler broadening (since the atoms will usually be at room temperature or above). This has several consequences. The first of these is that to adequately model these experiments the effects of Doppler broadening must be included. Moreover, in experiment the strong coupling fields must be intense enough to induce Rabi frequencies that exceed the Doppler widths (typically around $1 \mathrm{GHz}$ ) before any coherence effects (i.e., EIT) become apparent. Recent work [13] using focused lasers in near-two-photon Doppler-free conditions (using a ladder scheme in $\mathrm{Rb}$ ) has demonstrated that CW lasers can produce large enough Rabi couplings to observe transparency effects. In general, however, pulsed lasers with their far higher peak powers need to be used to induce sufficiently large Rabi couplings. In fact, by using pulsed lasers it becomes possible to avoid focusing of the beams altogether or to use weak focusing which eases the alignment problems in experiments and also permits the use of a plane-wave treatment for the phase matching (a treatment in terms of focused Gaussian beams would demand far more efforts due to the spatial variation in the susceptibilities that this would imply). As a consequence the laser linewidths will in general be nonnegligible, and will typically be larger than the natural line widths. Hence incorporation of laser linewidths into the treatment will become necessary. Although ours is a steady-state treatment of the problem, and hence only strictly applicable to $\mathrm{CW}$ laser experiments or to experiments in which the pulse duration is substantially larger than typical relaxation times, we believe the inclusion of real features (such as the laser bandwidth) will allow useful comparison to some features of experiments involving shorter pulses.

The paper is organized as follows. The outline of our model in Sec. II begins with the description of the dynamics of our system and transformation into a convenient matrix form. Then we investigate the susceptibility governing absorption, dispersion, and the nonlinear response. Results in the limit of no laser bandwidth or Doppler effects are presented for these susceptibilities. In the situation where both the driving Rabi frequency $\Omega_{d}$ and the pump Rabi frequency $\Omega_{p}$ are large we observe interesting behavior in the susceptibilities, indicating at certain combinations of laser field strengths there may be very large enhancements of the fourwave-mixing efficiency. In Sec. III the treatment is developed to include the effect of propagation and phase matching for the four-wave-mixing process, and in Sec. IV we extend our model by the inclusion of laser phase fluctuations and Doppler broadening. In Sec. V we present results under conditions matching experimentally feasible parameters (for pulsed laser experiments), the inclusion of phase fluctuations, and Doppler broadening. Details of the derivation of the phase-matching factor and of estimations of experimentally feasible parameters are given in Appendixes A and B, respectively.

\section{THE MODEL}

In this section we present a model describing the behavior of a three-level system under the influence of two applied fields (see Fig. 1). One of these fields (the "pump" field) couples the ground state $|c\rangle$ to excited state $|a\rangle$ via the twophoton Rabi frequency $\Omega_{p}$, the other field (the "driving" field) $\Omega_{d}$ couples states $|a\rangle$ and $|b\rangle$ via a single-photon process. We are primarily interested in the response of the system at the frequencies around the dipole allowed $|b\rangle-|c\rangle$ transition $\left(\omega_{l}\right)$. This model is developed on the assumption that the system has reached the steady state (i.e., the applied fields have no time dependence and have been switched on long enough for all initial transients to have vanished). We derive the equations for the density matrix and relate the matrix elements to the relevant susceptibilities. In this model we can incorporate various coherence degrading processes (e.g., spontaneous decay and collisions) and also (most importantly for many envisaged experiments) the effects of a finite laser bandwidth within the framework of the WienerLevy phase-diffusion model [14]. An important feature of our treatment is that we can treat the case of two strong fields (i.e., both $\Omega_{d}$ and $\Omega_{p}$ can be large) because we use the symbolic manipulation routine MATHEMATICA [15] to perform calculations that retain the effect of these couplings to all orders in the evaluation of the susceptibilities. 


\section{A. Dynamics}

\section{Equations of motion}

Here $\Omega_{i}$ denotes the Rabi frequency of the near resonance radiation, $\gamma_{i}$ the spontaneous emission rates, and $\Delta_{i}$ the detunings with $i$ corresponding to the various atomic levels. The corresponding Hamiltonian can be cast in the form

$$
H=H_{0}+V \text {, }
$$

where $H_{0}$ is the unperturbed Hamiltonian and $V$ the interaction Hamiltonian with

$$
\begin{aligned}
H_{0}= & \hbar \omega_{a}|a\rangle\left\langle a\left|+\hbar \omega_{b}\right| b\right\rangle\left\langle b\left|+\hbar \omega_{c}\right| c\right\rangle\langle c|, \\
V= & \hbar \Omega_{p} e^{-i 2 \omega_{p} t}|a\rangle\left\langle c\left|+\hbar \Omega_{d} e^{-i \omega_{d} t}\right| a\right\rangle\langle b| \\
& +\hbar \Omega_{l} e^{-i \omega_{l} t}|b\rangle\langle c|+\text { c.c. }
\end{aligned}
$$

with the electric dipole Rabi frequency $\Omega_{i j}$ defined via $\hbar \Omega_{i j}=\mu_{i j}\left|E\left(\omega_{i j}\right)\right|$ and $\left|E\left(\omega_{i j}\right)\right|$ being the electric field strength at the transition frequency $\omega_{i j}$. For the moment we take these fields to be monochromatic and assume all three fields are close to resonance, such that we can apply the rotating-wave approximation [16]. We obtain for the interaction Hamiltonian in the interaction picture

$$
\begin{aligned}
V^{\prime}= & \hbar \Omega_{p} e^{+i \Delta_{p} t}|a\rangle\left\langle c\left|+\hbar \Omega_{d} e^{+i \Delta_{d} t}\right| a\right\rangle\langle b| \\
& +\hbar \Omega_{l} e^{+i \Delta_{l} t}|b\rangle\langle c|+\text { c.c . }
\end{aligned}
$$

where $\Delta_{i}$ corresponds to the detunings given by

$$
\begin{gathered}
\Delta_{p}=\omega_{a c}-2 \omega_{p}, \\
\Delta_{d}=\omega_{a b}-\omega_{d}, \\
\Delta_{l}=\omega_{b c}-\omega_{l} .
\end{gathered}
$$

We now insert expression (4) for $V^{\prime}$ into the Liouville equation for the density matrix element evaluation,

$$
\begin{aligned}
\hbar \frac{\partial}{\partial t} \rho_{n m}(t)= & -i \sum_{k} H_{n k}(t) \rho_{k m}(t) \\
& +i \sum_{k} \rho_{n k}(t) H_{k m}(t)+\Lambda_{n m}
\end{aligned}
$$

(see [16]). Here $\Lambda_{n m}$ is a phenomenologically added decay term that corresponds to all the incoherent processes such as spontaneous decay, collisional broadening, etc. This leads to a set of nine coupled differential equations for $\rho_{a a}, \rho_{b b}, \rho_{c c}, \rho_{a b}, \rho_{a c}, \rho_{b c}, \rho_{b a}, \rho_{c a}$, and $\rho_{c b}$. We furthermore assume our system to be closed, i.e., $\rho_{a a}+\rho_{b b}+\rho_{c c}=1$, and thus reduce the number of equations by one. By moving into an appropriate rotating frame, the remaining time dependences can be conveniently eliminated:

$$
\begin{aligned}
& \rho_{a b}^{\prime}=\rho_{a b} e^{-i \Delta_{d} t}, \\
& \rho_{a c}^{\prime}=\rho_{a c} e^{-i \Delta_{p} t}, \\
& \rho_{b c}^{\prime}=\rho_{b c} e^{-i \Delta_{l} t},
\end{aligned}
$$

and similarly for the complex conjugates $\left(\rho_{b a}, \rho_{c a}\right.$, and $\left.\rho_{c b}\right)$. This removes the time dependence including terms of the form $e^{-i\left(\Delta_{p}-\Delta_{d}-\Delta_{l}\right) t}$ because the difference of detunings in the exponent cancels due to energy conservation. This leads us to the equations of motion,

$$
\begin{gathered}
\frac{\partial}{\partial t} \rho_{a a}=\frac{1}{2} i \Omega_{d}^{*} \rho_{a b}+\frac{1}{2} i \Omega_{p}^{*} \rho_{a c}-\frac{1}{2} i \Omega_{d} \rho_{b a}-\frac{1}{2} i \Omega_{p} \rho_{c a} \\
-\left(\gamma_{p}+\gamma_{d}\right) \rho_{a a}, \\
\frac{\partial}{\partial t} \rho_{b b}=-\frac{1}{2} i \Omega_{d}^{*} \rho_{a b}+\frac{1}{2} i \Omega_{l}^{*} \rho_{b c}+\frac{1}{2} i \Omega_{d} \rho_{b a}-\frac{1}{2} i \Omega_{l} \rho_{c b} \\
-\gamma_{l} \rho_{b b}+\gamma_{d} \rho_{a a}, \\
\frac{\partial}{\partial t} \rho_{a b}=\frac{1}{2} i \Omega_{d} \rho_{a a}-\frac{1}{2} i \Omega_{d} \rho_{b b}-i \Delta_{d} \rho_{a b}+\frac{1}{2} i \Omega_{l}^{*} \rho_{a c} \\
\quad-\frac{1}{2} i \Omega_{p} \rho_{c b}+\Lambda_{a b}, \\
\frac{\partial}{\partial t} \rho_{a c}=i \Omega_{p} \rho_{a a}+\frac{1}{2} i \Omega_{p} \rho_{b b}+\frac{1}{2} i \Omega_{l} \rho_{a b}-i \Delta_{p} \rho_{a c}-\frac{1}{2} i \Omega_{d} \rho_{b c} \\
\frac{\partial}{\partial t} \rho_{b c}=\frac{1}{2} i \Omega_{l} \rho_{a a}+i \Omega_{l} \rho_{b b}-\frac{1}{2} i \Omega_{d}^{*} \rho_{a c}-i \Delta_{l} \rho_{b c}+\frac{1}{2} i \Omega_{p} \rho_{b a} \\
-\frac{1}{2} i \Omega_{l}+\Lambda_{b c},
\end{gathered}
$$

where we have dropped the primes after the transformation and incoherent population relaxation terms have been added phenomenologically; see Fig. 1. The coherence damping coefficients $\Lambda_{i j}$ due to spontaneous emission and collision broadening are given by

$$
\begin{gathered}
\Lambda_{a b}=-\left\{\frac{1}{2}\left(\gamma_{d}+\gamma_{l}+\gamma_{p}\right)+\gamma_{a b}^{c o l}\right\} \rho_{a b}, \\
\Lambda_{a c}=-\left\{\frac{1}{2}\left(\gamma_{p}+\gamma_{d}\right)+\gamma_{a c}^{c o l}\right\} \rho_{a c}, \\
\Lambda_{b c}=-\left\{\frac{1}{2}\left(\gamma_{l}\right)+\gamma_{b c}^{c o l}\right\} \rho_{b c},
\end{gathered}
$$

where $\gamma_{i j}^{c o l}$ is the full width at half maximum Lorentzian collision width. In this paper we have ignored collisions, as in anticipated experiments, Doppler broadening and phase fluctuation broadening will dominate. The particular values we have assumed for the spontaneous emission rates in the following plots are those of krypton, i.e., $\gamma_{p}=0.11 \times 10^{6} \mathrm{rad}$ $\mathrm{s}^{-1}, \gamma_{d}=42.8 \times 10^{6} \mathrm{rad} \mathrm{s}^{-1}$, and $\gamma_{l}=225.7 \times 10^{6} \mathrm{rad} \mathrm{s}^{-1}$ [17]. This leads to $\Lambda_{a b}=-134.3 \times 10^{6}$ rad $\mathrm{s}^{-1} \rho_{a b}$, $\Lambda_{b c}=-112.85 \times 10^{6} \mathrm{rad} \mathrm{s}^{-1} \rho_{b c}$, and $\Lambda_{b c}=-21.5 \times 10^{6} \mathrm{rad}$ $\mathrm{s}^{-1} \rho_{a c}$.

\section{The Liouville superoperator $M$}

The observables that we are most interested in determining for our system are the absorption at the generated field 
$E\left(\omega_{l}\right)$, given by $\operatorname{Im}\left[\chi^{A}\right]$, the dispersion $\operatorname{Re}\left[\chi^{A}\right]$, and the nonlinear susceptibility governing $\chi^{F W}$, governing the process of four-wave mixing. Their precise definition and roles will be pointed out in the following; they are governed by the matrix elements of the density operator, which are too complicated to find through inspection. For convenience we express the equations of motion in terms of the real and imaginary parts of the matrix elements of the density operator, turning the $8 \times 8$ matrix into a $16 \times 16$ one. However, the imaginary parts of the populations are zero and the relations $\operatorname{Re}\left[\rho_{i j}\right]=\operatorname{Re}\left[\rho_{j i}\right]$ and $\operatorname{Im}\left[\rho_{i j}\right]=-\operatorname{Im}\left[\rho_{j i}\right]$ leaving us again with an $8 \times 8$ matrix. The final rate equation expressed in matrix form is

$$
\frac{\partial}{\partial t} R=M R+C
$$

where

$$
\left.R=\left(\begin{array}{c}
\operatorname{Re}\left[\rho_{a a}\right] \\
\operatorname{Re}\left[\rho_{b b}\right] \\
\operatorname{Re}\left[\rho_{a b}\right] \\
\operatorname{Im}\left[\rho_{a b}\right] \\
\operatorname{Re}\left[\rho_{a c}\right] \\
\operatorname{Im}\left[\rho_{a c}\right] \\
\operatorname{Re}\left[\rho_{b c}\right] \\
\operatorname{Im}\left[\rho_{b c}\right]
\end{array}\right) \text { and } C=\mid \begin{array}{c}
0 \\
0 \\
0 \\
0 \\
0 \\
-\frac{1}{2} \Omega_{p} \\
0 \\
-\frac{1}{2} \Omega_{l}
\end{array}\right)
$$

and

$M=\left(\begin{array}{cccccccc}-\left(\gamma_{p}+\gamma_{d}\right) & 0 & 0 & -\frac{1}{2}\left(\Omega_{d}+\Omega_{d}^{*}\right) & 0 & -\frac{1}{2}\left(\Omega_{p}+\Omega_{p}^{*}\right) & 0 & 0 \\ +\gamma_{d} & -\gamma_{l} & 0 & \frac{1}{2}\left(\Omega_{d}+\Omega_{d}^{*}\right) & 0 & 0 & 0 & -\frac{1}{2}\left(\Omega_{l}+\Omega_{l}^{*}\right) \\ 0 & 0 & \Lambda_{a b} & +\Delta_{d} & 0 & -\frac{1}{2} \Omega_{l}^{*} & 0 & -\frac{1}{2} \Omega_{p} \\ \frac{1}{2} \Omega_{d} & -\frac{1}{2} \Omega_{d} & -\Delta_{d} & \Lambda_{a b} & +\frac{1}{2} \Omega_{l}^{*} & 0 & -\frac{1}{2} \Omega_{p} & 0 \\ 0 & 0 & 0 & -\frac{1}{2} \Omega_{l} & \Lambda_{a c} & +\Delta_{p} & 0 & \frac{1}{2} \Omega_{d} \\ \Omega_{p} & \frac{1}{2} \Omega_{p} & \frac{1}{2} \Omega_{l} & 0 & -\Delta_{p} & \Lambda_{a c} & -\frac{1}{2} \Omega_{d} & 0 \\ 0 & 0 & 0 & \frac{1}{2} \Omega_{p} & 0 & \frac{1}{2} \Omega_{d}^{*} & \Lambda_{b c} & +\Delta_{l} \\ \frac{1}{2} \Omega_{l} & \Omega_{l} & \frac{1}{2} \Omega_{p} & 0 & \frac{1}{2} \Omega_{d}^{*} & 0 & -\Delta_{l} & \Lambda_{b c}\end{array}\right)$.

Note that from our definition of the Rabi frequency there is no distinction between $\Omega_{i}$ and $\Omega_{i}^{*}$; however, there is a physical distinction: $\Omega_{i}$ relates to excitation processes and $\Omega_{i}^{*}$ to deexcitation. Although there is a slight loss in clarity caused by suppressing this distinction (the final expressions for $\rho_{i j}$ will contain terms of the form $\Omega_{i} \Omega_{i}$ rather than $\Omega_{i} \Omega_{i}^{*}=\left|\Omega_{i}\right|^{2}$ ), there is no change in the final outcome.

Implicit in this treatment is that the density matrix elements are now time-independent parameters (the steady-state assumption). Hence this treatment cannot deal with the case of fields evolving rapidly compared to the relaxation rates of the system. However, a number of recent experiments (e.g., [9] and our own work) do use pulsed lasers which are not necessarily long compared to the relaxation times. In these experiments the pulse duration (typically a few nanoseconds) usually satisfies the condition $\tau_{\text {pulse }}>>1 / \Omega_{d}$, but the other conditions necessary for a time independent treatment to be valid are not satisfied [e.g., usually $\tau_{\text {pulse }} \sim 1 / \Omega_{p}$ or $1 / \gamma$ (the natural width)]. Nonetheless we can use this model to treat the case of $\mathrm{CW}$ and long pulse experiments and to 
make contact with some features of the short pulsed laser case through the inclusion of finite laser linewidth effects (see Sec. IV).

\section{B. The susceptibilities}

\section{Relation between the density matrix element and the susceptibility}

The response of a medium to an electric field is governed by its polarization

$$
\begin{aligned}
\langle P\rangle= & N \operatorname{Tr}[\mu \rho] \\
= & N\left(\mu_{a b} \rho_{b a}+\mu_{b a} \rho_{a b}+\mu_{a c} \rho_{c a}+\mu_{c a} \rho_{a c}\right. \\
& \left.+\mu_{b c} \rho_{c b}+\mu_{c b} \rho_{b c}\right),
\end{aligned}
$$

where $N$ denotes the number density of atoms with dipole moment $\mu$. Each of the three terms above relates to the contribution of the polarization close to a particular frequency: the first to $\omega_{a b}$, the second to $\omega_{a c}$, and the last, the one we are interested in, to $\omega_{b c} \approx \omega_{l}$. So, we have $P\left(\omega_{l}\right)=N\left(\mu_{b c} \rho_{c b}+\mu_{c b} \rho_{b c}\right)$. The two terms here relate to the two amplitudes in the complex exponential formulation of the fields. Thus, we arrive at the following relation between the real amplitude of the polarization and the density matrix element $\rho_{b c}$ :

$$
P\left(\omega_{l}\right)=2 N \mu_{b c} \rho_{c b} .
$$

The form of $\rho_{b c}$ which we obtain from solving the equation of motion of the density matrix elements in steady state is rather complicated and not instructive at first glance. If we express $\rho_{b c}$ as a power expansion in the Rabi frequencies (i.e., the fields) in a perturbative approach, then each term corresponds to a definite physical process involving the absorption and emission of photons (see Fig. 2). Although a calculation involving only low-order processes would not be satisfactory in the limit of large $\Omega$ 's, this figure does show how the amplitudes of different order processes can interfere to cause cancellation of the absorption.

Turning to the linear contribution of the susceptibility we recall from solving Maxwell's equations in a linear medium

$$
P_{l i n}\left(\omega_{l}\right)=\epsilon_{0} \chi^{A}\left(\omega_{l}\right)\left(E_{d}, E_{p}\right) E_{l},
$$

where $\chi^{A}\left(\omega_{l}\right)\left(E_{d}, E_{p}\right)$ denotes the susceptibility linear in the field $E_{l}$ but up to all orders in the other fields $E_{d}$ and $E_{p}$ with

$$
\chi^{A}\left(\omega_{l}\right) E_{l}=\left(2 N \mu_{b c} / \epsilon_{0}\right) \rho_{b c l i n} .
$$

Considering Eq. (20) we notice that the terms of $\rho_{b c}$ which are linear in the field $\omega_{l}$, i.e., linear in $\Omega_{l}$ contribute to the linear susceptibility. If we expand $\rho_{b c}$ in powers of $\Omega_{l}$ we obtain

$$
\begin{aligned}
\rho_{b c}= & A\left(\Omega_{d}, \Omega_{p}, \gamma_{i j}, \Delta_{i}\right) \\
& +B\left(\Omega_{d}, \Omega_{p}, \ldots\right) \Omega_{l}+C(\cdots)\left|\Omega_{l}\right|^{2}+\ldots
\end{aligned}
$$

It is worth pointing out that due to our simplification $\Omega_{i}=\Omega_{i}^{*}$ the term $B$ in the above refers to both absorption and emission of a photon at $\omega_{l}$, and indeed gives the overall effect at $\omega_{l}$ be it absorption or emission. The higher-order

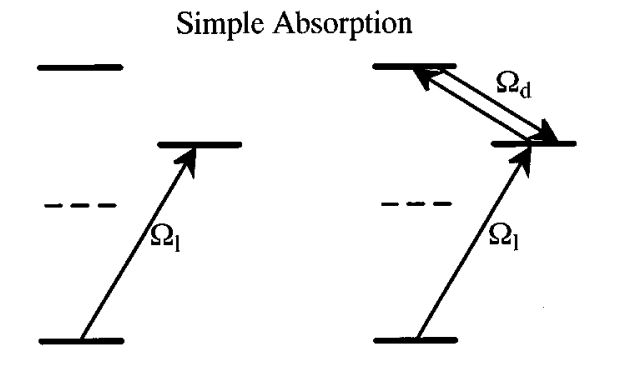

Terms linear in $\Omega_{1} \quad$ Terms linear in $\Omega_{1}\left|\Omega_{d}\right|^{2}$

Four-Wave Mixing


Terms linear in $\Omega_{\mathrm{p}} \Omega_{\mathrm{d}}^{*} \quad$ Terms linear in $\left|\Omega_{\mathrm{p}}{ }^{2} \Omega_{\mathrm{p}} \Omega_{\mathrm{d}}\right|^{2} \Omega_{\mathrm{d}}^{*}$

FIG. 2. Interfering processes which contribute to the susceptibility on the transition of interest $b-c$. The two lower figures indicate forward mixing to first and higher orders. We are interested in additional nonlinear processes due to extra "loops" on the $a-b$ and $a-c$ transitions.

odd terms in $\Omega_{l}$ will be relevant to nonlinear absorption and dispersion. These can be ignored provided $\Omega_{l}$ is small. The even-order terms relate to processes that couple the levels $|b\rangle$ and $|c\rangle$ together without a direct (nonreturning) transition between the two. These will be discussed later in the context of $\chi^{F W}$. So, Eq. (20) can be rewritten as

$$
B\left(\Omega_{d}, \Omega_{p}, \gamma_{i j}, \Delta_{i}\right) \Omega_{l}=\frac{2 N \epsilon_{0}}{\mu_{b c}} \chi^{A}\left(\omega_{l}\right) E_{l} .
$$

Now there is a simple relation between the coupling dipole field and the Rabi frequency $\hbar \Omega_{i j}=\mu_{i j}\left|E_{i}\right|$ :

$$
B\left(\Omega_{d}, \Omega_{p}, \gamma_{i j}, \Delta_{i}\right) \Omega_{l}=\frac{2 N \epsilon_{0}}{\mu_{b c}} \times \frac{-\hbar}{\mu_{b c}} \times \chi^{A} \Omega_{l} .
$$

This then yields

$$
\chi^{A}=-\frac{2 N \mu_{b c}^{2}}{\epsilon_{0} \hbar} B\left(\Omega_{d}, \Omega_{p}, \gamma_{i j}, \Delta_{i}\right)
$$

or

$$
\chi^{A}=-\frac{6 N \pi c^{3}}{\omega_{l}^{3}} A_{c b} B\left(\Omega_{d}, \Omega_{p}, \gamma_{i j}, \Delta_{i}\right),
$$

where $A_{c b}$ is the Einstein A coefficient associated with the transition $b-c . \chi^{A}$ and $B$ are of course complex quantities (the real part giving dispersion and the imaginary part giving absorption). 


\section{Nonlinear susceptibility}

For the evaluation of $\chi^{A}$ we have considered terms linear in the generated field $E_{l}$. Assuming the conventional form of the lowest-order nonlinear susceptibility $\chi^{(3)}$, we need restrict ourselves to terms linear in $E_{p}^{2} E_{d}$ or $\Omega_{p} \Omega_{d}^{*}$ (since $\left.\Omega_{p} \propto E_{p}^{2}\right)$. This would give us $\chi^{(3)}\left(-\omega_{l}, \omega_{p}, \omega_{p},-\omega_{d}\right)$. However, these are not the only terms corresponding to the physical processes we are interested in. We would like to incorporate terms of all orders in the driving field $\Omega_{d}$ (and the pump $\Omega_{p}$ ), and in fact all these higher-order terms con- tribute to the coherent effect of electromagnetically induced transparency. This means we also need to include, e.g., $\chi^{(5)}\left(-\omega_{l}, \omega_{p}, \omega_{p},-\omega_{d}, \omega_{d},-\omega_{d}\right)$ and $\quad \chi^{(7)}\left(-\omega_{l}, \omega_{p}\right.$, $\left.\omega_{p},-\omega_{d}, \omega_{d},-\omega_{d}, \omega_{d},-\omega_{d}\right)$ and terms to all orders in $\Omega_{d}$ and $\Omega_{p}$, and if we want to include $\Omega_{l}$ to all orders, we need include all even-order terms in $\Omega_{l}$ of the expansion in Eq. (21). This gives rise to a quantity that is not the conventional third-order susceptibility $\chi^{(3)}$ but a term that is proportional to the sum of the susceptibilities multiplied by their fields, i.e.,

$$
\begin{aligned}
A\left(\Omega_{d}, \Omega_{p}, \gamma_{i j}, \Delta_{i}\right)+C(\cdots)\left|\Omega_{l}\right|^{2}+\cdots \propto & \chi^{(3)}\left(-\omega_{l}, \omega_{p}, \omega_{p},-\omega_{d}\right) E_{p}^{2} E_{d}+\chi^{(5)}\left(-\omega_{l}, \omega_{p}, \omega_{p},-\omega_{d}, \omega_{d},-\omega_{d}\right) E_{p}^{2} E_{d}^{3} \\
& +\chi^{(7)}\left(-\omega_{l}, \omega_{p}, \omega_{p},-\omega_{d}, \omega_{d},-\omega_{d}, \omega_{d},-\omega_{d}\right) E_{p}^{2} E_{d}^{5}+\chi^{(5)}\left(-\omega_{l}, \omega_{p},-\omega_{p}, \omega_{p},\right. \\
& \left.-\omega_{p},-\omega_{d}\right) E_{p}^{4} E_{d}+\cdots
\end{aligned}
$$

which is here referred to as the field coupling strength. The field generated at $\omega_{l}$ with Rabi frequency $\Omega_{l}$ can still be considered small compared to the Rabi frequencies $\Omega_{d}$ and $\Omega_{p}$, so that our main interest is in the term $A$ in Eq. (21). This term $A$ cannot strictly be associated with any singleorder susceptibility of the right-hand side of above Eq. (26) as $A$ includes all nonlinearities in $\Omega_{d}$ and $\Omega_{p}$. The lowest order term of $A$ is obviously $\chi^{(3)}\left(-\omega_{l}, \omega_{p}, \omega_{p},-\omega_{d}\right)$ so that we define, in agreement with the convention in the literature, the susceptibility responsible for four-wave mixing as

$$
\chi^{F W}:=\kappa \frac{A}{\Omega_{d} \Omega_{p}} .
$$

Here we need still emphasize that $A$ and $\chi^{F W}$ remain functions of $\Omega_{d}$ and $\Omega_{p}$. The constant $\kappa$ can be estimated by substituting $P\left(\omega_{l}\right)=\epsilon_{0} \chi^{3}\left(-\omega_{l}, \omega_{p}, \omega_{p},-\omega_{d}\right) E_{d} E_{p}^{2}$ into Eq. (18). This yields

$$
\kappa=\frac{4}{\epsilon_{0} \hbar} \mu_{a b} \mu_{b c} \sum_{i} \frac{\mu_{a i} \mu_{i c}}{\hbar\left(\omega_{i c}-\omega_{p}\right)}
$$

which cannot be calculated precisely due to the lack of empirical data for the atomic dipole matrix elements $\mu_{i c}$, where the sum over $i$ means the inclusion of all levels $i$ of the atom. The variable $\chi^{F W}$ is therefore evaluated in arbitrary (but of course always the same) units throughout the paper.

\section{Results for susceptibilities with no Doppler and laser linewidths}

The results shown in this section were obtained using the model presented so far and so include neither the laser linewidth effects nor Doppler broadening (dealt with in Sec. IV). Furthermore no propagation effects (phase matching) are included (see Sec. III). They are useful as a guide to the real optical properties of a medium by giving the susceptibility (and hence the absorption, dispersion, and nonlinear response) of a single-atom in a perfectly monochromatic field. In particular they can be used to reveal at which detunings
$\Delta_{l}$ and under what combinations of coupling strengths $\Omega_{p}$ and $\Omega_{d}$ the single-atom response may show enhanced $\chi^{F W}$ and at the same time vanishing $\operatorname{Im} \chi^{A}$ (or even gain). It is useful in the monochromatic limit to calculate the singleatom response as a pointer to any interesting parameter regimes that might be investigated in more detail in subsequent theoretical work in which laser linewidths, Doppler broadening, and phase matching are included and also as a guide to interesting experiments.

In Fig. 3 we show the results for quantities $\operatorname{Im}\left[\chi^{A}\right], \operatorname{Re}\left[\chi^{A}\right]$, and $\left|\chi^{F W}\right|$ as functions of the detuning $\Delta_{l}$ in the case of typical atomic parameters (taking those of the $\mathrm{Kr}$ scheme enumerated in Appendix B) with a small pump field $\Omega_{p} \approx 0$ and a modest Rabi frequency of the driving field $\Omega_{d}=2.5 \times 10^{9} \mathrm{rad} \mathrm{s}^{-1}$. This was chosen to be ten times larger than the radiative decay term $\gamma_{b c}$. We clearly reproduce the well-known results [8] already reported for this limit, i.e., that at zero $\Delta_{l}$ we have low absorption, zero dispersion, and a still resonantly enhanced $\left|\chi^{F W}\right|$. This is evidence that we would expect an enhancement in the field generated at $\omega_{l}$ (compared to the weak field limit) in a scheme operated with these parameters. The values of $\chi^{A}$ in these plots are the single-atom values (multiplied by a factor of $10^{23}$ ) and for a many-atom system we multiply these by the number density $N_{\mathrm{Kr}}$ to obtain the total linear susceptibility. $\left|\chi^{F W}\right|$ is displayed in arbitrary units; however, the scaling factor is identical for all the results presented in this paper, so the magnitude can be compared under different conditions. The widths of the Autler-Townes split peaks in the absorption curve [Fig. 3(a)] are determined by the radiative widths of the atom.

It is interesting to look at the behavior of one of these absorption peaks (located near $\Delta_{l}= \pm \Omega_{d} / 2$ ) as a function of $\Omega_{p}$ as it is increased to larger values and at the same time when a stronger driving field is used, i.e., $\Omega_{d}=2.5 \times 10^{10} \mathrm{rad}$ $\mathrm{s}^{-1}$. This is shown in Fig. 4. As $\Omega_{p}$ is increased the absorption peak decreases and eventually crosses below zero at a value of $\Omega_{p} \sim 2 \times 10^{8} \mathrm{rad} \mathrm{s}^{-1}$ to show gain (positive values) rather than absorptive loss at $\Delta_{l}=\Omega_{d} / 2$. This is, we believe, 
(a)

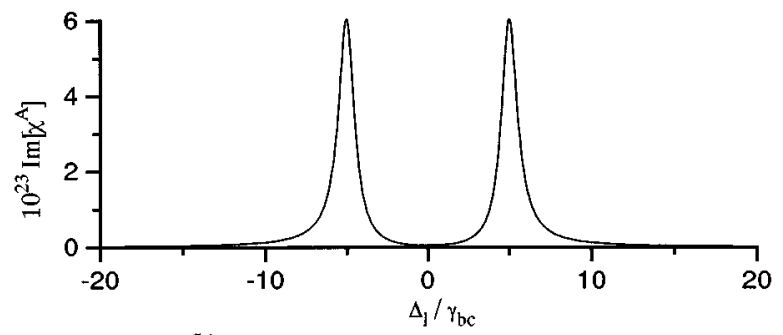

(b)
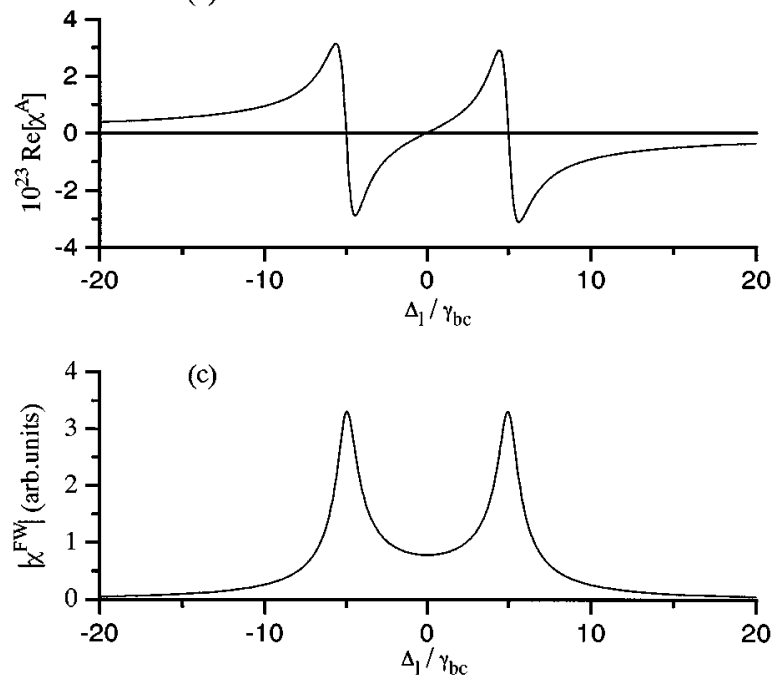

FIG. 3. The variables $\operatorname{Im}\left[\chi^{A}\right], \operatorname{Re}\left[\chi^{A}\right]$, and $\left|\chi^{F W}\right|$ in the parameter range of a $\mathrm{Kr}$ atom with a small pump field $\Omega_{p} \approx 0$ and a modest Rabi frequency of the driving field $\Omega_{d}=16 \mathrm{GHz}$ $=10 \gamma_{b c}$. These results are similar to the previous results [8], which were restricted to the linear regime in the pump Rabi frequency.

due to Raman pumping of population into the dressed state associated with $|b\rangle$ (while the absorption becomes close to zero).

In these conditions the population in state $b$, $\rho_{b b}\left(\Delta_{l}, \Omega_{p}\right)$, has reached significant values (at $\Delta_{l}=\Omega_{d} / 2$ it reaches a value of $\sim 0.1$ for $\Omega_{p}=2 \times 10^{8} \mathrm{rad} \mathrm{s}^{-1}$ ); see Fig. 5 . We furthermore note that the separation of the two roots asymptotically approaches the value of $\Omega_{p}$. The splitting of the absorption peak into a doublet is clearly visible and can be understood as a second Autler-Townes splitting of the Autler-Townes satellites already "dressed" by $\Omega_{d}$, with a large gain region in the center. At the center frequency of these split features the absorption becomes very low (or vanishes) and this coupled to the population in $|b\rangle$ leads to the appearance of gain. This can be interpreted as gain without inversion in the sense that we are in the regime where in the bare atom basis $\rho_{b b}<\rho_{c c}$ (Fig. 5). The equivalent graphs for $\left|\chi^{F W}\right|$, which are not presented here, show saturation and a broadening of the peak, rather similar to the behavior of $\operatorname{Im}\left[\chi^{A}\right]$ but with no negative region. We now consider the dependence on the detuning $\Delta_{l}$ of the susceptibilities in the situation where both driving fields are large. These are displayed in Fig. 6 as a function of the ratio $\Omega_{p} / \Omega_{d}$ for $\Omega_{d} / \gamma_{b c}=100$. We see a complex behavior displaying a number of features: at low values of $\Omega_{p} \sim 0$ the behavior shows the usual induced transparency at $\Delta_{l}=0$ with absorption peaks separated by exactly $\Omega_{d}$. As $\Omega_{p}$ is increased into the

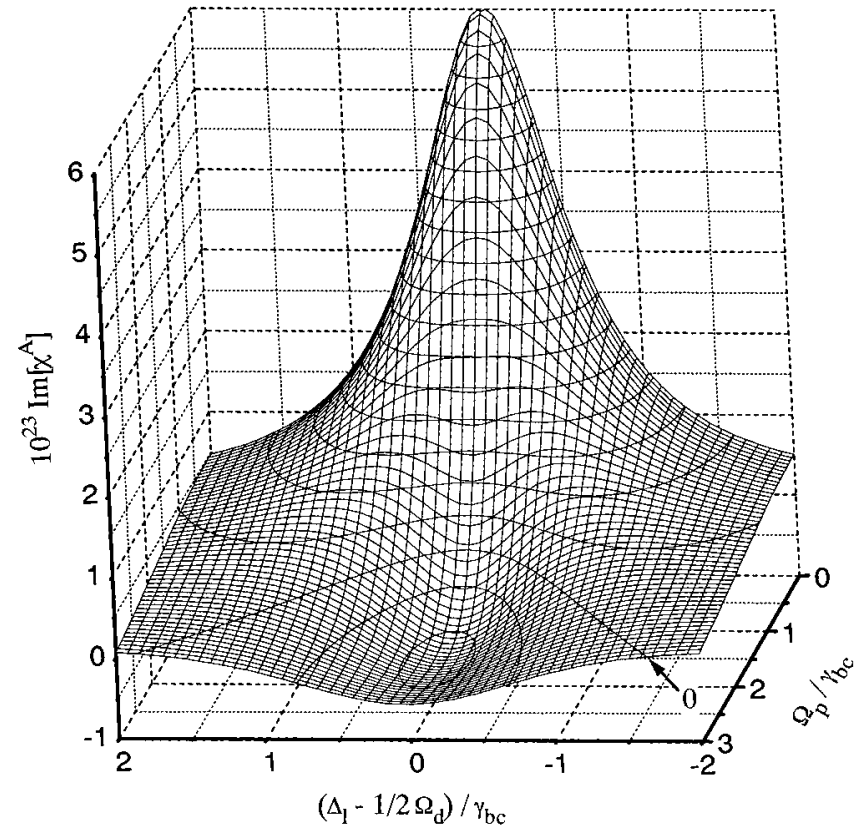

FIG. 4. The linear absorption $\operatorname{Im}\left[\chi^{A}\right]$ as a function of the detuning $\Delta_{l}$ around a single Rabi sideband and the pump field Rabi frequency $\Omega_{p}$ for $\Omega_{d}=160 \mathrm{GHz}=100 \gamma_{b c}$.

range $0.1<\Omega_{p} / \Omega_{d}<0.25$ the absorption maxima develop regions with gain at their centers bounded by regions of higher absorption (i.e., the behavior presented already in Fig. 4). In this regime there are in effect four absorption peaks, the outer pair separated by a region of actual gain and the inner pair by a region of very low absorption (centered on $\left.\Delta_{l}=0\right)$. For larger $\Omega_{p}$ the four peak structure and gain regions are broadened and merge to form a single broad central gain region spanning the frequency range $\pm \Omega_{d} / 2$. The refractive index features $\operatorname{Re}\left[\chi^{A}\right]$ show a parallel behavior, evolving through the characteristic double dispersion structure of the usual transparency range to develop additional peaks in the parameter range $0.1<\Omega_{p} / \Omega_{d}<0.25$ to become a broad single dispersion profile type structure at larger values of $\Omega_{p}$. In both cases the eventual broadening of the structures for large $\Omega_{p}$ can be interpreted as power broadening of the spectral features. The nonlinear susceptibility also develops the four peaked structure in the same parameter range and it too shows "saturation" at higher $\Omega_{p}$. It should be noted that although $\left|\chi^{F W}\right|$ does decline dramatically it remains above zero at $\Delta_{l}=0$ (except near $\Omega_{p} / \Omega_{d}=1$, where interference effects seem probable) even for large $\Omega_{p}$. Destructive interference is evident, however, beginning at the centers of the Autler-Townes satellites and resulting in zeros in $\left|\chi^{F W}\right|$ at $\Delta_{l} \sim \pm 25 \gamma_{b c}$ for $\Omega_{p}=\Omega_{d}$.

Although the behavior becomes more complicated when two strong fields are present, the features are interpretable in terms of the dressing of a three-level system by two strong fields. Other work on similar systems which examined spontaneous emission and absorption of strongly driven threelevel systems [18] considers similar behavior. In our case the second strong field $\left(\Omega_{p}\right)$ splits the Autler-Townes satellites (due to $\Omega_{d}$ ) eventually driving them to a position such that the system can have a large response even at $\Delta_{l}=0$ thus severely modifying the systems response at these values. We 
(a)

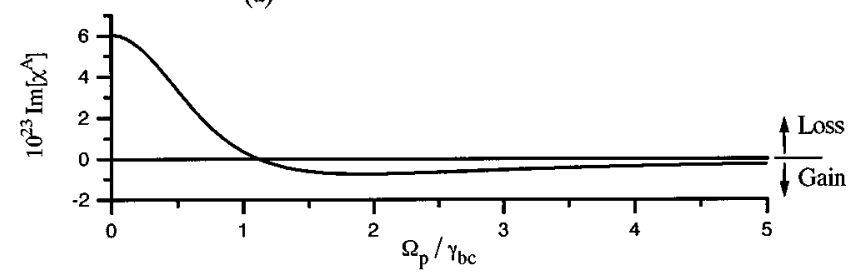

(b)

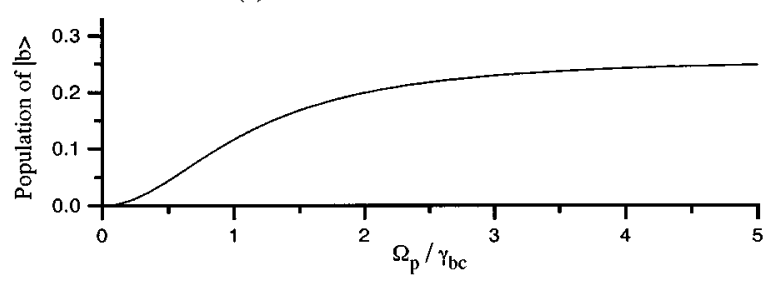

(c)

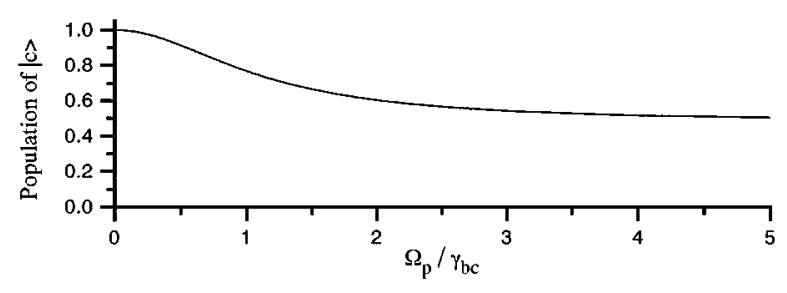

FIG. 5. The absorption $\operatorname{Im}\left[\chi^{A}\right]$ (a) and excited state population of level $b$ (b) and ground-state population of level $c$ (c). The parameters employed are $\Omega_{d}=100 \gamma_{b c}$ and $\Delta_{l}=0$.

note that the authors of [18] have developed a dressed-state interpretation for the emission and absorption spectrum of an arbitrary bichromatically driven three-level system in the limit of at least one intense driving field. Although their paper has not considered the nonlinear response, their approach is of value in understanding the behavior we observe. In particular, they attribute the existence of gain regions to the interplay between ordinary stimulated emission, absorption, and spontaneous decay processes with the Raman transitions induced among the atomic levels by the strong driving fields. As opposed to Ref. [18], we consider the spectrum not on the transitions on which the system is strongly driven, and the quantum dressed states need involve the photon number of all three fields. Any photon of one field can be exchanged by a combination of the other two fields which leads to additional degeneracies of the bare states and thus to a more complex picture than that of three dressed states for each set of photon numbers which are separated by an equal amount of energy.

The discussion of the implication of the behavior of susceptibilities on the efficiency of four-wave mixing is deferred to the end of the next section in which phase matching will be included. For large values of $\Omega_{p}$ there appears to be a "saturation" of the susceptibilities, with broadening of distinct spectral features, hence $\left|\chi^{F W}\right|$ is greatly reduced from the values encountered if $\Omega_{p}$ were small. This does not, however, necessarily mean that the four-wave-mixing efficiency is small since, as will be seen in the next section, if phase matching is included the conversion efficiency depends upon $\left|\chi^{F W}\right| /\left|\chi^{A}\right|$, which can become large if the linear susceptibility is close to zero even for small values of the nonlinear susceptibility. The presence of gain on the $|b\rangle-|c\rangle$ transition in the single-atom response implies laser type growth of the field at $\omega_{l}$. We will discuss the possible implications of this in Sec. V.

\section{FOUR-WAVE MIXING WITH EIT: INCLUSION OF PHASE MATCHING}

\section{A. Efficiency of four-wave mixing}

To calculate the intensity generated in a nonlinear wavemixing scheme we must, in addition to the computation of the single-atom responses (given here by the susceptibilities), calculate the effects of propagation through an extended medium composed of many atoms interacting with the fields. Therefore the next task is to solve Maxwell's equations in a nonlinear, homogeneous, dielectric material in order to include the effects of propagation and phase matching on the nonlinear optical conversion efficiency. To do this we make the following assumptions.

(i) The slowly varying envelope approximation. This means expressing the field as the product of two components, one of which oscillates at the optical frequency, and the other whose time variations occur on a much slower time scale: $E(r, z, t)=\frac{1}{2}\left[A(r, z, t) e^{-i(\omega t-k z)}+\right.$ c.c. $]$ and is valid for our laser fields. The atomic polarization is then proportional to the field strength $A(r, z, t)$.

(ii) $\chi_{N L}$ is independent of time. This is not always a satisfactory approximation in resonant nonlinear interactions in the case of time-dependent pulses since there may be significant changes occurring in the excited state populations during the pulse, but we are constrained to making this timeindependent approximation in order to make our calculations tractable.

(iii) The group velocity is weakly dependent on $\omega$, i.e., the phase-matching condition is constant over the linewidth.

(iv) The nonlinear interactions are solely due to the electric dipole interaction, i.e., we can ignore the magnetic polarization $M$.

We then obtain

$$
\nabla_{\perp}^{2} A_{j}(r, z, t)+2 i k_{j} \frac{\partial}{\partial z} A_{j}(r, z, t)=4 \pi \omega_{j}^{2} P_{j}^{N L} e^{-i \Delta k_{j} z}
$$

where $A_{j}$ is the field amplitude at $\omega_{j}, \nabla_{\perp}$ is the gradient operator in cylindrical polar coordinates perpendicular to $z$, $P_{j}^{N L}$ is the nonlinear polarization at $\omega_{j}$, and $\Delta k_{j}$ is the wave-vector mismatch $\Delta k_{j}=k_{j}-k_{j}^{p}$ for the nonlinear process coupling fields to $\omega_{j}$ (see [19]). The above is in Gaussian units. The $P^{N L}$ for our system is given by

$$
P^{N L}\left(\omega_{l}\right)=\frac{1}{4} \chi^{F W} A_{p}^{2} A_{d}^{*}
$$

After inserting Eq. (30) into (29) we make further assumptions.

(i) The driving fields will be treated as constant in amplitude over distance $z$ and the nonlinear contributions to $A_{d}$ and $A_{p}$ are insignificant. Here we are ignoring any changes in $A_{d}$ and $A_{p}$ due to the four-wave-mixing process. 


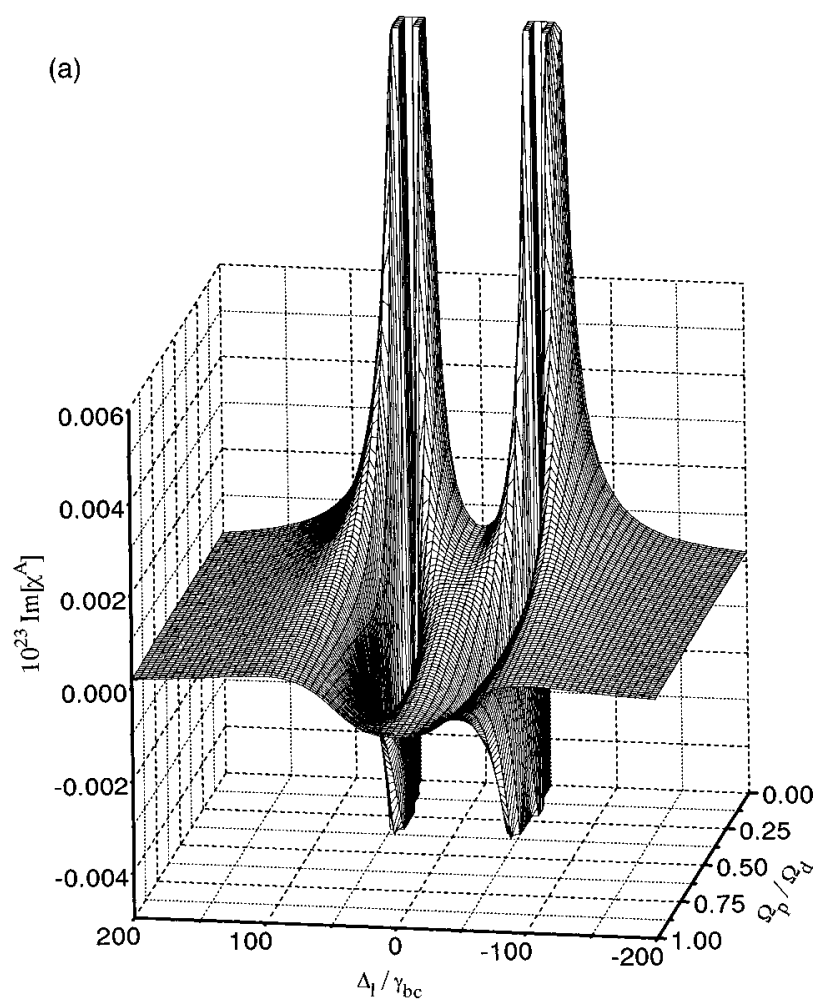

(b)
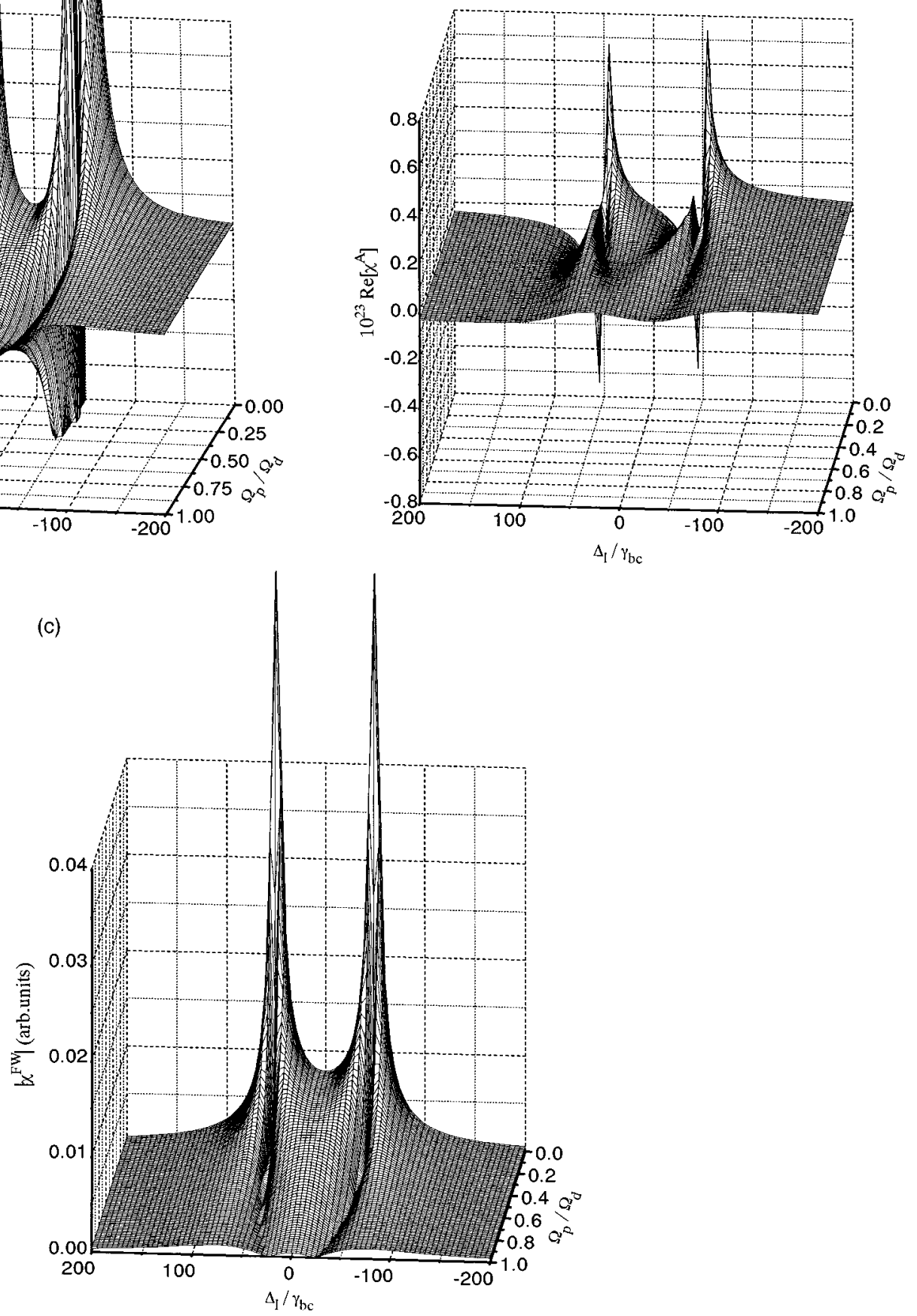

FIG. 6. The absorption $\operatorname{Im}\left[\chi^{A}\right]$ (a), the dispersion $\operatorname{Re}\left[\chi^{A}\right]$ (b), and the nonlinear susceptibility $\left|\chi^{F W}\right|$ (c) in the parameter range of a $\mathrm{Kr}$ atom as a function of the detuning $\Delta_{l}$ and the ratio $\Omega_{p} / \Omega_{d}$ for $\Omega_{d}=100 \gamma_{b c}$. These results extend the linear parameter regime in the pump Rabi frequency of Ref. [8].

(ii) Plane-wave geometry, i.e., a nonfocused geometry.

(iii) Uniform number density in the mixing medium. This ensures that $\chi^{F W}$ is independent of $z$.

This leads to

$$
\frac{\partial}{\partial z} A_{l}=i \frac{\pi^{2}}{n\left(\omega_{l}\right) \lambda_{l}} \chi^{F W} A_{p}^{2} A_{d} e^{-i \Delta k_{l} z},
$$

where

$$
\Delta k_{l}=k_{l}+k_{d}-2 k_{p} .
$$

Equation (29) describes the rate of change of $A_{l}$ due to the nonlinear four-wave-mixing process, ignoring the absorption and dispersion at $\omega_{l}$. This needs to be included 
because we are close to resonance. If we do include these terms, Eq. (31) (now in S.I. units) becomes

$$
\begin{aligned}
\frac{\partial}{\partial z} A_{l}= & i \frac{\omega_{l}}{4 c} \chi^{F W} A_{p}^{2} A_{d} e^{-i \Delta k_{l} z}-\frac{\omega_{l}}{2 c} \operatorname{Im}\left[\chi^{A}\right] A_{l} \\
& +i \frac{\omega_{l}}{2 c} \operatorname{Re}\left[\chi^{A}\right] A_{l} .
\end{aligned}
$$

This is a simple first-order linear differential equation which can be solved to give $A_{l}$. The boundary condition chosen here is $A_{l}(z=0)=0$. This can be related to the intensity by

$$
I_{j}=\frac{2 n}{Z_{0}}\left|E_{j}\right|^{2}=\frac{2 n}{Z_{0}} A_{j}^{2}
$$

where $Z_{0}$ is the impedance of free space. This calculation has been done to yield (see Appendix A)

$$
I\left(\omega_{l}\right)=\frac{3 n \omega_{l}^{2}}{8 Z_{0} c^{2}}\left|\chi^{F W}\right|^{2}\left|A_{p}\right|^{4}\left|A_{d}\right|^{2} \frac{\left[1+e^{-\left(\omega_{l} / c\right) \operatorname{Im}\left[\chi^{A}\right] z}-2 e^{-\left(\omega_{l} / 2 c\right) \operatorname{Im}\left[\chi^{A}\right] z} \cos \left(\left\{\Delta k+\left(\omega_{l} / 2 c\right) \operatorname{Re}\left[\chi^{A}\right]\right\} z\right)\right]}{\left(\omega_{l}^{2} / 4 c^{2}\right) \operatorname{Im}\left[\chi^{A}\right]^{2}+\left\{\Delta k+\left(\omega_{l} / 2 c\right) \operatorname{Re}\left[\chi^{A}\right]\right\}^{2}} .
$$

It must be noted here that the contribution to the dispersion from the $\omega_{l}$ transition is treated separately from the other contributions due to its rapid variation close to resonance; $k_{l}$ in Eq. (32) is the wave vector at $\omega_{l}$ due to all the other transitions apart from the $\omega_{l}$ transition.

It is interesting to note how this compares with the ratio $R$ discussed in [8]. Now if we ignore all phase mismatch due to the other transitions, i.e., $\Delta k=0$, and take the terms that affect the amplitude of $I_{l}$ rather than its $z$ dependence we obtain

$$
I\left(\omega_{l}\right) \propto \frac{\left|\chi^{F W}\right|^{2}}{\left|\chi^{A}\right|^{2}} \propto \frac{|A|^{2}}{\left|\chi^{A}\right|^{2}\left|\Omega_{p}\right|^{2}\left|\Omega_{d}\right|^{2}}=R^{\prime 2}
$$

rather than

$$
I\left(\omega_{l}\right) \propto \frac{\left|\chi^{F W}\right|^{2}}{\left[\operatorname{Im} \chi^{A}\right]^{2}} \propto \frac{|A|^{2}}{\left[\operatorname{Im} \chi^{A}\right]^{2}\left|\Omega_{p}\right|^{2}\left|\Omega_{d}\right|^{2}}=R^{2}
$$

which was proposed in [8]. The parameter $R$ proposed by Harris was derived by making more restrictive assumptions, i.e., that the phase mismatch due to $\Delta k$ was zero (by addition of a suitable quantity of buffer gas) and that the dispersive influence on the generated intensity due to $\operatorname{Re}\left[\chi^{A}\right]$ was also negligible (by limiting the calculation to exact resonance). $R^{\prime}$ thus represents a useful figure of merit that can parametrize the four-wave-mixing efficiency for arbitrary detunings. In the following we will consider this parameter to investigate four-wave mixing under the influence of electromagnetically induced transparency.

\section{B. Propagation over finite lengths}

As discussed above, the intensity generated via four-wave mixing as a function of the phase mismatch $\Delta k$ is given by Eq. (34). We can separate the $z$ dependence from Eq. (34); this is conveniently expressed in terms of an amplitude multiplied by an oscillatory factor:

$$
\begin{aligned}
I \propto & I_{o}\left[1+e^{-\left(\omega_{l} / c\right) \operatorname{Im}\left[\chi^{A}\right] z}\right. \\
& \left.-2 e^{-\left(\omega_{l} / 2 c\right) \operatorname{Im}\left[\chi^{A}\right] z} \cos \left(\left\{\Delta k+\left(\omega_{l} / 2 c\right) \operatorname{Re}\left[\chi^{A}\right]\right\} z\right)\right],
\end{aligned}
$$

where

$$
I_{o}=\frac{\left|\chi^{F W}\right|^{2}\left|A_{p}\right|^{4}\left|A_{d}\right|^{2}}{\left(\omega_{l}^{2} / 4 c^{2}\right)\left|\chi^{A}\right|^{2}+\Delta k^{2}+\left(\omega_{l} / 2 c\right) \Delta k \operatorname{Re}\left[\chi^{A}\right]} .
$$

With no phase mismatch $(\Delta k=0)$ and replacing $\left|\chi^{F W}\right|^{2}\left|A_{p}\right|^{4}\left|A_{d}\right|^{2}$ with $|A|^{2}$ as discussed above we have

$$
I_{o} \propto \frac{|A|^{2}}{\left|\chi^{A}\right|^{2}} \equiv R^{\prime 2} \Omega_{p}^{2} \Omega_{d}^{2}
$$

In order to point out the relation between $R^{\prime}$ and the generated electric field at $\omega_{l}$ we need to investigate the oscillatory term more closely. We define this term as $f$ :

$$
\begin{aligned}
f= & 1+e^{-\left(\omega_{l} / c\right) \operatorname{Im}\left[\chi^{A}\right] z} \\
& -2 e^{-\left(\omega_{l} / 2 c\right) \operatorname{Im}\left[\chi^{A}\right] z} \cos \left(\left\{\Delta k+\left(\omega_{l} / 2 c\right) \operatorname{Re}\left[\chi^{A}\right]\right\} z\right)
\end{aligned}
$$

making the coordinate transformation

$$
\begin{gathered}
z^{\prime}=\frac{\omega_{l}}{2 c} \operatorname{Im}\left[\chi^{A}\right] z, \\
C=\frac{\left(\operatorname{Re}\left[\chi^{A}\right]+\left(2 c / \omega_{l}\right) \Delta k\right)}{\operatorname{Im}\left[\chi^{A}\right]} .
\end{gathered}
$$

This simplifies to

$$
f=1+e^{-2 z^{\prime}}-2 e^{-z^{\prime}} \cos \left(C z^{\prime}\right) .
$$

\section{Four-wave mixing in the limit of no laser linewidth or Doppler broadening}

From the discussion in Sec. III A we saw that the singleatom contribution to generating a field at $\omega_{l}$ can be approximated by $R^{\prime}=\left|\chi^{F W}\right| /\left|\chi^{A}\right|$ (or as has been used in the literature, $R=\left|\chi^{F W}\right| / \operatorname{Im}\left[\chi^{A}\right]$ ). In Fig. 7 we have displayed $R$ and $R^{\prime}$ for the same parameters as Fig. 3 (i.e., $\left.\Omega_{d}=10 \gamma_{b c} \approx 7 \times 10^{9} \mathrm{rad} \mathrm{s}^{-1}, \Omega_{p} \approx 0\right)$. Concerning $\left|\chi^{F W}\right|$ the units remain arbitrary due to the lack of knowledge of the matrix element on the two-photon pump transition (but of 



FIG. 7. The effectivity of four-wave mixing, as measured by $R$ (a) and $R^{\prime}$ (b) as a function of the laser field detuning on the $b-c$ transition for the same parameters as Fig. 3 (i.e., $\Omega_{d}=10 \gamma_{b c} \approx 16$ $\mathrm{GHz}, \Omega_{p} \approx 0$ ).

course is held constant throughout this paper). Since the $\operatorname{Re}\left[\chi^{A}\right]$ is identically zero at $\Delta_{l}=0$, the values of $R$ and $R^{\prime}$ will be equal at this position. The form of $R^{\prime}$ is similar to reported experimental results (e.g., see [10]) whereas $R$, because it does not include contributions to the phase matching of $\operatorname{Re}\left[\chi^{A}\right]$, is not strictly valid for $\Delta_{l} \neq 0$.

If we assume that $R^{\prime}\left(\Delta_{l}=0\right)=R\left(\Delta_{l}=0\right)$ determines the amplitude of the field generated at $\omega_{l}$ we can use this quantity to investigate how the generation efficiency at resonance is related to $\Omega_{d}$ and $\Omega_{p}$ within a regime where only $\Omega_{d}$ is strong. We have investigated $R\left(\Delta_{l}=0\right)$ versus $\Omega_{d}$ and $\Omega_{p}$ with $1<\Omega_{d}<30 \mathrm{THz}, 1<\Omega_{p}<10 \mathrm{MHz}$. We find that the value is essentially constant over the entire range once $\Omega>\gamma$, and since generated intensity $E_{l}$ is proportional to $\left(R \Omega_{p} \Omega_{d}\right)^{2}$ we have a linear increase of $E_{l}$ in the two Rabi frequencies.

If we allow the two driving fields to be strong such that $\Omega_{p} \approx \Omega_{d}>>\gamma_{b c}$ (see susceptibilities plotted in Fig. 6), then we obtain results for the generation efficiency $R^{\prime}$ shown in Fig. 8. In this plot the value of $R^{\prime}$ in arbitrary units is shown as a function of the ratio $\Omega_{p} / \Omega_{d}$ over the range $\Delta_{l}= \pm 100 \gamma_{b c}$ for a value of $\Omega_{d}=100 \gamma_{b c}$. When $\Omega_{p}$ is small the single narrow peak structure centered at $\Delta_{l}=0$ (as in Fig. 7) is seen. This corresponds to a small $\operatorname{Im}\left[\chi^{A}\right]$ and large $\left|\chi^{F W}\right|$ at $\Delta_{l}=0$ while the zero value of $\operatorname{Re}\left[\chi^{A}\right]$ gives optimal phase matching. At larger values of $\Omega_{p}$ the nonlinear susceptibility $\left|\chi^{F W}\right|$ decreases, however so does $\operatorname{Im}\left[\chi^{A}\right]$ (even becoming negative), hence the ratio $R^{\prime}$ increases at

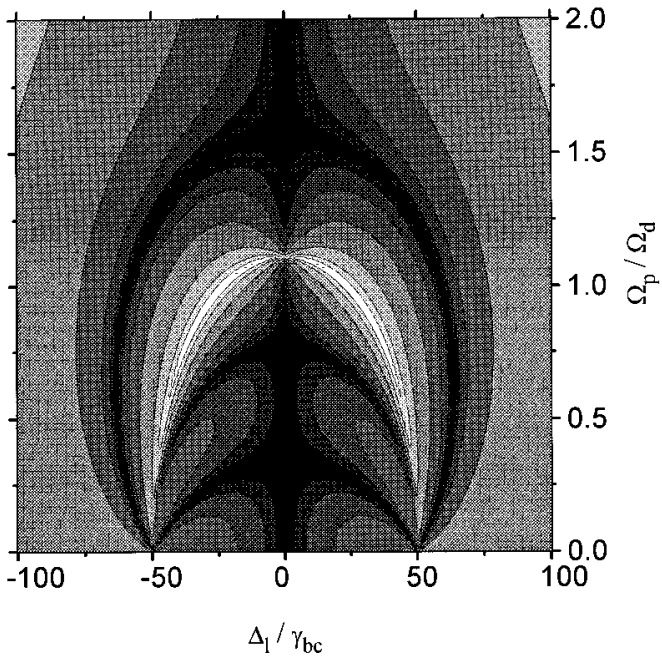

FIG. 8. Contour representation of the effectivity of four-wave mixing, as measured by $R^{\prime}$ as a function of $\Delta_{l}$ and the ratio $\Omega_{p} / \Omega_{d}$ with $\Omega_{d}=100 \gamma_{b c}$ in arbitrary units on a logarithmic scale.

$\Delta_{l}=0$ reaching a peak at $\Omega_{p} / \Omega_{d} \sim 0.4$. Here it seems that $\left|\chi^{A}\right|$ has vanished, and the $R^{\prime}$ becomes very large. This occurs again when the ratio reaches $\sim 3.4$. There is, however, a difficulty in interpreting $R^{\prime}$ when there is gain present $\left(\operatorname{Im}\left[\chi^{A}\right]<0\right)$ in the system, i.e., when $\Omega_{p}>0.25$ the field at $\omega_{l}$ would be expected to depend on the source term $\rho_{b c}$ but to grow exponentially and not to depend on phase matching. It would seem reasonable that since $\rho_{b c}$ is in our treatment (up to orders linear in $\Omega_{l}$ ) given by $A+B$ in Eq. (21), both $\left|\chi^{A}\right|$ and $\left|\chi^{F W}\right|$ contribute to the generated field. When gain is present the situation is therefore changed as the four-wave mixing will still be parameterized by $R^{\prime}$ (the efficiency depends on $\left|R^{\prime}\right|^{2}$ ) but there will also be an exponential growth of the field with $z$ due to the presence of this gain [see Eq. (34)]. A situation in which both four-wave mixing and gain are operating simultaneously is clearly unusual (a situation in which stimulated emission competes with fourwave mixing at separate frequencies is, however, discussed in the literature [20]), but this appears to be the case in this regime. This point will be returned to in the next section.

We also present results that explore the spatial $z$ dependence of the intensity of the field at $\omega_{l}$. This output field as well as depending on $R^{\prime}\left(\Omega_{p} \Omega_{d}\right)^{2}$ also depends on the phase-matching factor $f$ introduced in Eqs. (41) and (44). In Fig. 9 we find $f$ vs $z^{\prime}$ for different values of $C$. $C$ as defined in Eq. (43) is essentially the ratio of total phase mismatch to absorption: small values indicate that the coherence length is much larger than the absorption length and large values show that the opposite is true. In the former case we would expect little dephasing of the propagation field over the length over which the intensity grows, while in the latter we would anticipate seeing the effects of much dephasing (i.e., interference). This is indeed seen in the results. We can see that after $z^{\prime}>4$ there is very little $z$ dependence on the intensity, and that for very small $z$, the intensity is approximately a sinusoidal function of $z$, proportional to $R^{\prime}$. The intensity as a function of the distance $z$ will be displayed later when laser linewidth effects are incorporated. 
(a)

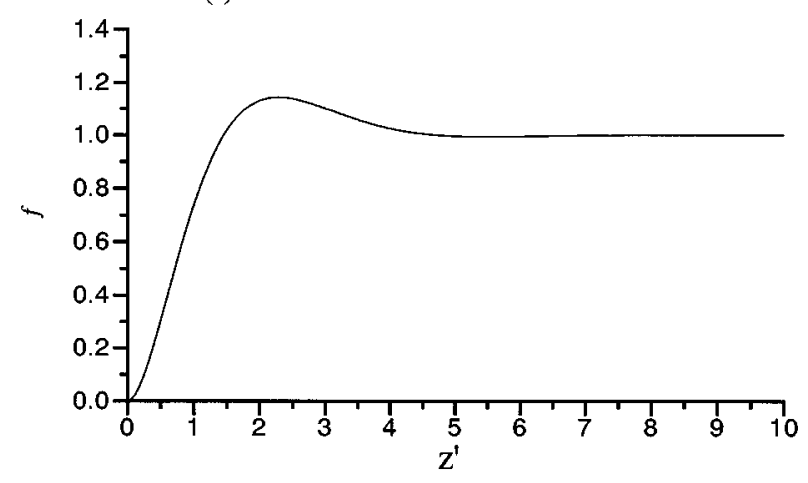

(b)

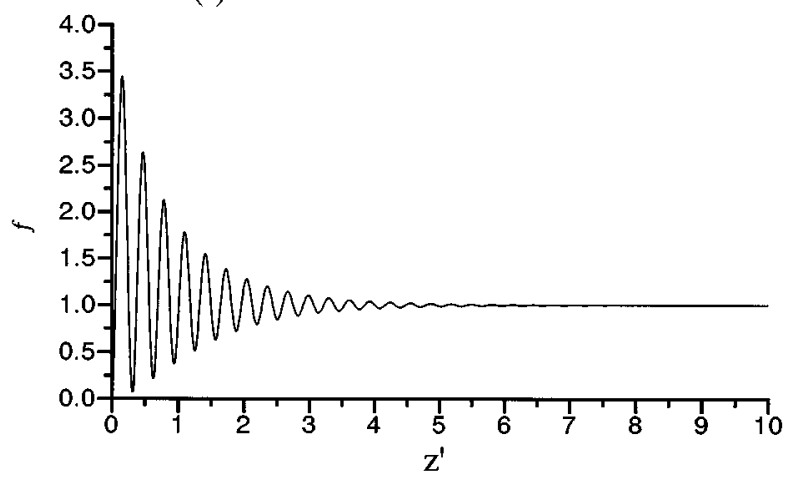

FIG. 9. The effect of phase mismatch as indicated by the function $f$ for $C=1$ (a) and $C=20$ (b).

\section{TREATMENT INCLUDING REALISTIC LINEWIDTHS}

An experimental investigation of four-wave mixing inevitably involves lasers with a nonvanishing linewidth (even in CW experiments). In a gas cell setup Doppler broadening will also be important. We will now consider these problems, respectively, neglecting the effect of collision broadening which is small compared to the other broadening mechanisms for the range of parameters in which we are presently interested.

\section{A. Influence of the laser linewidths}

So far we have assumed purely monochromatic radiation. This means that the largest incoherent decay in the system is due to spontaneous emission. A typical value for this would be about $10 \mathrm{MHz}$. Now the experimental realization of large Rabi frequencies requires the use of pulsed lasers, with typical bandwidths of up to $1 \mathrm{GHz}$. In order to appreciate how laser bandwidths could effect coherent processes it is useful to adopt the following model. The fluctuating nature of laser light can be thought of as a monochromatic source with a degree of phase diffusion and amplitude diffusion (e.g., [21]). Since the coherence between two levels depends on the relative phases of the different order processes, we would expect the coherence to be strongly affected by this diffusion. This is not the case for the populations as here the amplitude fluctuations are superimposed on a large constant value and are hence much less important.

The effects of phase diffusion can be simply added to our model if we use the Wiener-Levy model [14]. In this, frequency fluctuations are regarded as zero-mean Gaussian
Markoff stochastic processes; this gives rise to a Lorentzian line shape in the power spectrum. However it is at variance with the essentially Gaussian profile encountered for most lasers. Since a Lorentzian profile has significantly larger values at large detunings from the center frequency than a Gaussian of the same width, it is worth bearing in mind that we will probably be overestimating the effects of laser linewidths at large detunings. Similar treatments are reported in the literature [22], but these are not then incorporated into treatments that include realistic phase matching or the possibility of two strong fields.

The addition of the stochastic term into the Hamiltonian of the lasers affects the equations of motion of the density matrix elements (see [23]) by the addition of a decay term in the off-diagonal elements only. If we go back to Eq. (13) and add the laser bandwidths we obtain

$$
\begin{gathered}
\Lambda_{a b}=-\frac{1}{2}\left(\gamma_{d}+\gamma_{l}+\gamma_{p}\right) \rho_{a b}-\gamma_{l w a b} \rho_{a b}, \\
\Lambda_{a c}=-\frac{1}{2}\left(\gamma_{p}+\gamma_{d}\right) \rho_{a c}-\gamma_{l w a c} \rho_{a c} \\
\Lambda_{b c}=-\frac{1}{2} \gamma_{l} \rho_{b c}-\gamma_{l w b c} \rho_{b c} .
\end{gathered}
$$

From the literature (see $[24,25]$ ) we find the following expressions for $\gamma_{l w i j}$ :

$$
\begin{gathered}
\gamma_{l w a b}=\gamma_{l w d} \\
\gamma_{l w a c}=\gamma_{l w p 1}+\gamma_{l w p 2}+\gamma_{l w p 1 p 2} \\
\gamma_{l w b c}=\frac{1}{2}\left(\gamma_{l w a b}+\gamma_{l w a c}+\gamma_{l w a b a c}\right)
\end{gathered}
$$

which is true when the coherence $\rho_{a c}$ is driven predominantly by the two-photon pump (with negligible contributions from the fields at $\omega_{d}$ and $\omega_{l}$ ).

It is easy to see the origin of (46) where $\gamma_{l w d}$ is the linewidth of the strong driving field. The form of $\gamma_{l w a c}$ in (47) is due to the fact that the $|a\rangle \rightarrow|c\rangle$ transition is of twophoton nature. Here $\gamma_{l w p 1}$ refers to the linewidth of the laser transition to the virtual half-way state, $\gamma_{l w p 2}$ to the laser up to the two-photon resonance, and the $\gamma_{l w p 1 p 2}$ is due to the possible correlation of the two lasers phase fluctuations,

$$
\begin{aligned}
& \text { if } p 1 \text { and } p 2 \text { are the same laser } \gamma_{l w p 1 p 2}=2 \gamma_{l w p} \text {, } \\
& \text { if } p 1 \text { and } p 2 \text { are uncorrelated } \gamma_{l w p 1 p 2}=0 \text {, } \\
& \text { if } p 1 \text { and } p 2 \text { are anticorrelated } \gamma_{l w p 1 p 2}=-2 \gamma_{l w p} \text {. }
\end{aligned}
$$

We can see that if the lasers were truly anticorrelated the contribution of the linewidth of the pump laser can be eliminated. Normally $p 1$ and $p 2$ will be due to the same laser, so $\gamma_{l w a c}=4 \gamma_{p}$. The extra $\gamma_{\text {lwabac }}$ term in Eq. (48) is due to a similar possible correlation, however the two lasers responsible for $\gamma_{p}$ and $\gamma_{d}$ will almost always be uncorrelated so this term will be set to be equal to zero. So, to summarize, the most common schemes will have 


$$
\begin{gathered}
\Lambda_{a b}=-\frac{1}{2}\left(\gamma_{d}+\gamma_{l}+\gamma_{p}+2 \gamma_{l w d}\right) \rho_{a b}, \\
\Lambda_{a c}=-\frac{1}{2}\left(\gamma_{p}+\gamma_{d}+8 \gamma_{l w p}\right) \rho_{a c}, \\
\Lambda_{b c}=-\frac{1}{2}\left(\gamma_{l}+\gamma_{l w d}+4 \gamma_{l w p}\right) \rho_{b c} .
\end{gathered}
$$

\section{B. Incorporation of Doppler broadening}

Another broadening mechanism we need to include at finite temperatures is the Doppler spread in the frequencies seen by the atoms (even for a monochromatic field). This we deal with numerically by performing an appropriately weighted sum of the results of the calculation of susceptibilities over all the possible Doppler induced detunings. Let us take $v$ as positive when traveling in the same direction as the driving laser beams, the $x$ axis. As the atoms have thermal velocities we only consider velocities in the direction of the laser beam (all second-order Doppler effects are ignored). The frequency seen by an atom with velocity $v$ is given (when $v \ll c$ ) by

$$
\omega(v) \cong(1-\beta) \omega_{v=0},
$$

where $\beta=v / c$. If this is substituted into the definition of the detunings given in [7], then we obtain

$$
\begin{gathered}
\Delta_{p}(v)=\Delta_{p}+2 \beta \omega_{p}, \\
\Delta_{d}(v)=\Delta_{d}+\beta \omega_{d}, \\
\Delta_{l}(v)=\Delta_{l}+\beta \omega_{l} .
\end{gathered}
$$

The normalized 1D Doppler velocity distribution is

$$
d N\left(v_{x}\right)=N_{0}\left(M / 2 \pi k_{b} T\right)^{\frac{1}{2}} e^{-\left(M / 2 k_{b} T\right) v_{x}^{2}} d v_{x},
$$

where $N_{0}$ is the number density, $M$ the mass, $k_{b}$ Boltzmann's constant, and $T$ the absolute temperature. The problem is now treated numerically. In order to obtain a plot of some function $f\left(\Omega_{d}, \Omega_{p}, \gamma_{i j}, \Delta_{i}(v)\right)$ against some variable $a$ including Doppler broadening the procedure chosen here is described as follows: (i) Determine $f$ at a (large) number of points within the velocity spread for some value of $a$; (ii) multiply each value of $f$ by the appropriate weighting for each velocity point; (iii) the sum of these contributions is the Doppler averaged value of $f$ at that particular $a$.

The appropriate weighting factor for a given $v$ is the integral of Eq. (40) between $v+d v$ and $v-d v$ where $2 N d v$ is the total velocity spread included. If we express the distribution in terms of the root-mean-square velocity $v_{r m s}$,

$$
d N\left(v_{x}\right)=N_{0} / \sqrt{2 \pi} v_{r m s} e^{-\frac{1}{2} v_{x}^{2} / v_{r m s}^{2}} d v_{x}
$$

then the integral between $v_{x 1}$ and $v_{x 2}$ is given by

$$
\Delta N\left(v_{x 1}, v_{x 2}\right)=\frac{1}{2} N_{0} \operatorname{Erf}\left[\frac{1}{2} v_{x 2} / v_{r m s}\right]-\operatorname{Erf}\left[\frac{1}{2} v_{x 1} / v_{r m s}\right],
$$

where $\operatorname{Erf}[]$ refers to the error function.

\section{Four-wave mixing with realistic linewidths: Results and discussion}

In this section we discuss the main results, including the influence of the various broadening mechanisms. Since the process of electromagnetically induced transparency has been proposed as a way to enhance the nonlinear mixing in a resonant four-wave-mixing scheme for the efficient generation of coherent VUV radiation, we will look at a typical VUV scheme (sum-difference frequency mixing in krypton $[26,27])$. In fact, the frequency of the two-photon transition (providing the coupling $\Omega_{p}$ ) is in this case in the UV, and to achieve efficient two-photon excitation (needed to operate this scheme) pulsed lasers are in practice the only option. We are of course constrained to using our steady-state model. We will be particularly concerned with the effects of laser linewidth, Doppler broadening, and phase matching on the frequency up conversion. A discussion of the feasible parameter values in a realistic experiment is given in Appendix B. Here we use the values of these parameters to calculate the likely behavior of the system including our generalized treatment of the single-atom susceptibilities (with strong driving fields and laser linewidths) along with Doppler broadening and phase matching.

Unfortunately because of the short wavelength of $\omega_{p}$ and the necessity to generate it via frequency doubling even using a pulsed laser, the two-photon Rabi frequency $\left(\Omega_{p}\right)$ in this scheme will be relatively small, typically $\sim 60 \mathrm{KHz}$, as a maximum in an unfocused geometry (see Appendix A), and therefore not greater than the Doppler width, so the condition of two strong fields cannot be reached in this particular scheme (except by tight focusing). However, for alternative schemes in alkali-metal vapors (e.g., $\mathrm{Li}$ ) in which the twophoton wavelength is in the visible or near IR range much larger $\Omega_{p}$ values can be reached and the two strong fields case accessed. Our calculations are performed using the $\mathrm{Kr}$ atomic parameters, but these are representative to those encountered in other atomic systems (e.g., Li), and hence are still a useful guide as to what may be achieved experimentally.

\section{Effects of laser linewidths on the behavior with a strong driving field}

The addition of the laser linewidths in the off-diagonal density matrix elements will increase the magnitude of the incoherent decays substantially relative to the radiative decays. The strong driving field has a linewidth of $500 \mathrm{MHz}$, and the pump field a frequency doubled $\left(\lambda_{p}=212.55 \mathrm{~nm}\right)$ width of $2.5 \mathrm{GHz}$. The typical radiative decays are of the order of $10 \mathrm{MHz}$. Here we are interested in how the linewidths effect the magnitude of the absorption, dispersion, and nonlinear susceptibility. Shown in Fig. 10 are the absorption, dispersion, and $\left|\chi^{F W}\right|$ for the regime in which $\gamma_{l w d}=500 \mathrm{MHz}, \gamma_{l w d}=0, \gamma_{l w}=2.5 \mathrm{GHz}$. Assuming spontaneous decay rates of krypton, we then obtain $\gamma_{a b}=3.28 \times 10^{9} \mathrm{rad} \mathrm{s}^{-1}=5.21 \times 10^{8} \mathrm{~Hz}, \gamma_{b c}=3.31 \times 10^{10}$ $\mathrm{rad} \mathrm{s}^{-1}=5.27 \times 10^{9} \mathrm{~Hz}$, and $\gamma_{a c}=6.29 \times 10^{10} \mathrm{rad} \mathrm{s}^{-1}$ $=1.00 \times 10^{10} \mathrm{~Hz} . \Omega_{d}$ is set to ten times $\gamma_{a c}$ which is the largest of the incoherent decays due to the large bandwidth 
(a)



(b)

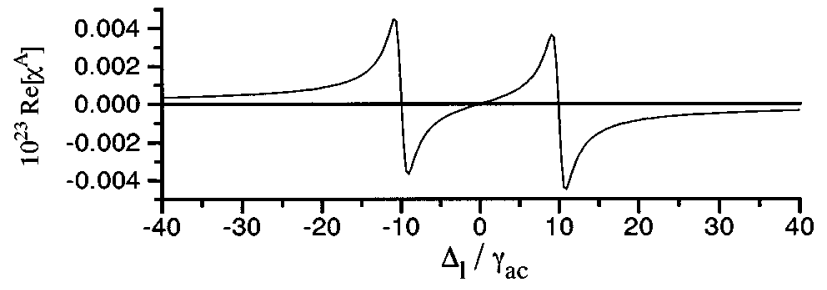

(c)

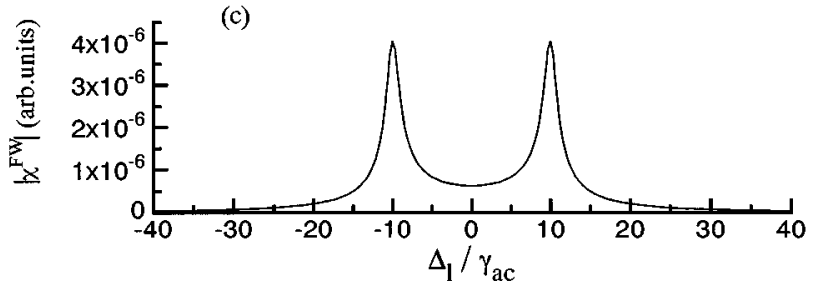

FIG. 10. The absorption $\operatorname{Im}\left[\chi^{A}\right]$ (a), the dispersion $\operatorname{Re}\left[\chi^{A}\right]$ (b), and the nonlinear susceptibility $\left|\chi^{F W}\right|$ (c) under the influence of broadening mechanism with $\gamma_{l w d}=500 \mathrm{MHz}, \gamma_{l w p}=2.5 \mathrm{GHz}$; $\gamma_{\text {Doppler }}=0.7 \gamma_{a c}$ as in krypton at room temperature and $\Omega_{d}=20 \gamma_{a c}$.

of the pump laser (and because this is a two-photon transition) and $\Omega_{p}$ is small in these plots. Importantly the destructive interference effects on the absorption cannot survive the inclusion of such large laser linewidths. We see, however, that the general form of the curves remains similar to those in Fig. 3. The low absorption and vanishing dispersion at resonance remain even with the inclusion of laser linewidths. This, however, is at the cost of increasing the driving Rabi frequency by two orders of magnitude so the resonant enhancement in the susceptibility is very reduced. Furthermore, the actual magnitudes of all the susceptibilities are substantially altered compared with those of Fig. 3.

We now use the results of Sec. III to translate these altered susceptibilities (including laser linewidths) into generated field intensities. These results are shown in Fig. 11, where a calculation involving the full phase-matching factor, in addition to laser and Doppler linewidths, has been performed. In the plots shown there is no absolute intensity scale (due to uncertainties in the magnitude of $\left|\chi^{F W}\right|$ ), but the relative magnitude between plots is preserved to enable comparisons to be made. In these plots $\Omega_{d}=10^{12} \mathrm{rad} \mathrm{s}^{-1}$ and $\Omega_{p}=6 \times 10^{6} \mathrm{rad} \mathrm{s}^{-1}$ (comparable to the conditions in Fig. 10). The intensity is plotted as a function of laser detuning and of path length for a gas density of 1 mbar. Two alternative detuning scenarios are considered. Figure 11(a) has $\omega_{d}$ remaining fixed and resonant and $\omega_{p}$ tuned. Figure
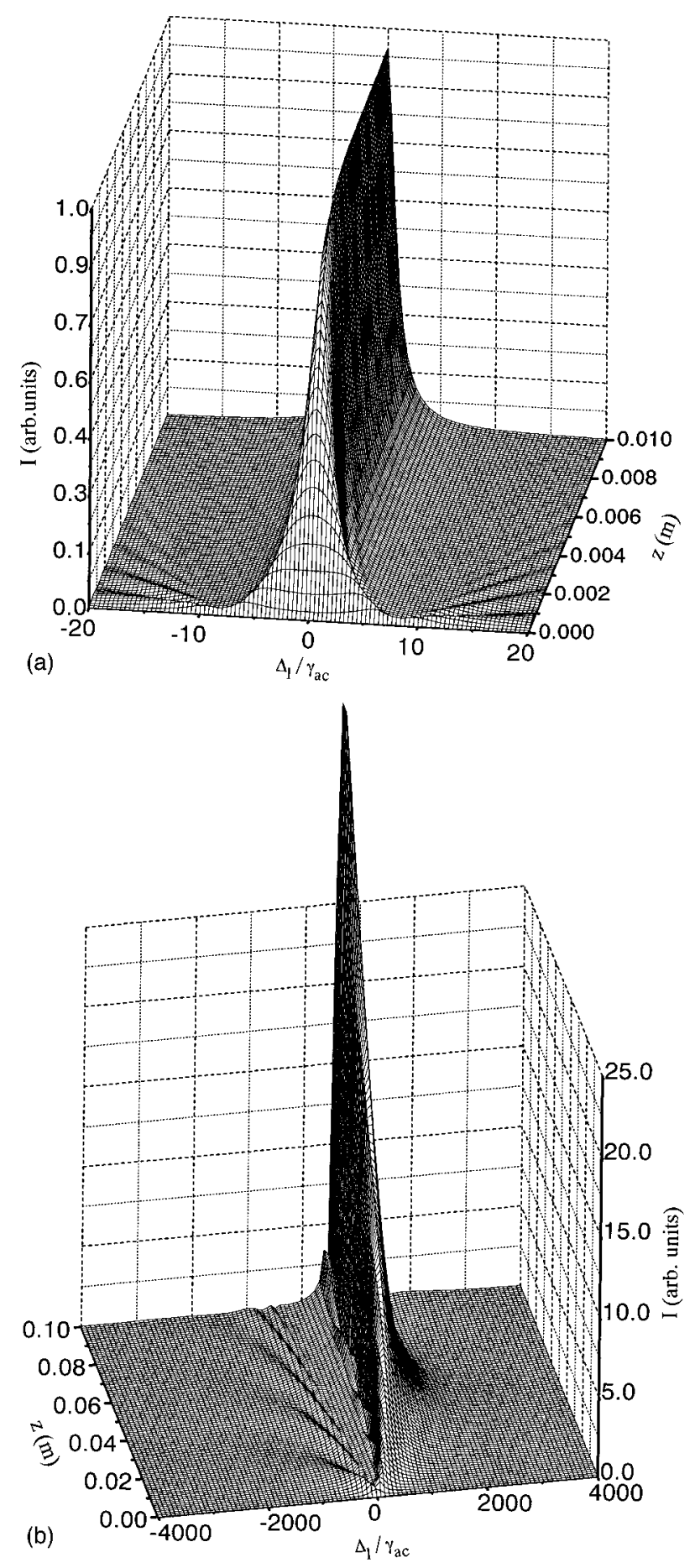

FIG. 11. The observable intensity $I$ as a function of the detuning $\Delta_{l}$ for (a) $\Delta_{d}=0$ and (b) $\Delta_{p}=0$ and in both cases the distance $z$. The parameters used are $\gamma_{l w d}=500 \mathrm{MHz}, \gamma_{l w p}=2.5 \mathrm{GHz}, \Omega_{d}=10^{12} \mathrm{rad}$ $\mathrm{s}^{-1}, \Omega_{p}=610^{6} \mathrm{rad} \mathrm{s}^{-1}$, and a pressure of $p=1 \mathrm{mbar}$ has been assumed.

11(b) has $\omega_{d}$ tunable and $\omega_{p}$ fixed and in two-photon resonance. In Fig. 11(a) a strong growth in intensity is only encountered for laser detunings close to resonance $\Delta_{l}=0$, and elsewhere the growth in the field intensity shows a damped oscillatory behavior. In this case the growth saturates on resonance after a path length of $2 \mathrm{~mm}$. Figure 11(b) indicates a different situation in which the driving field is operated far from resonance: in this case more efficient four-wave mixing 


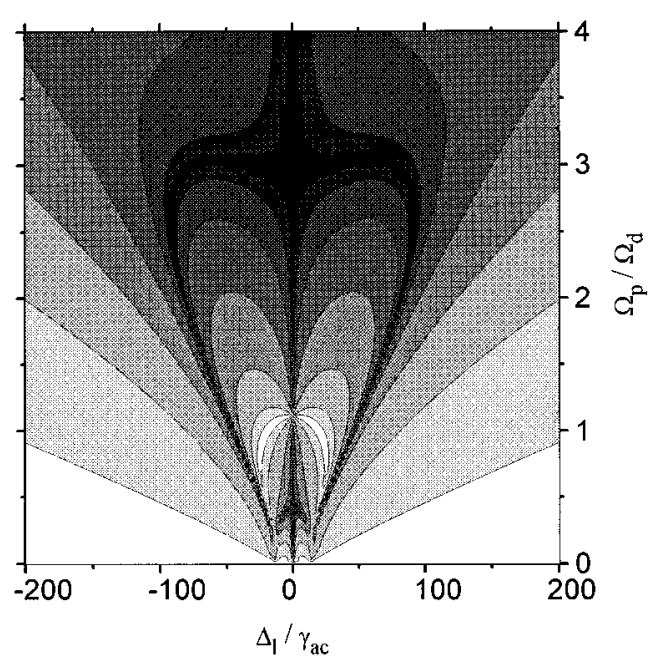

FIG. 12. Contour representation of the effectivity of four-wave mixing, as measured by $R^{\prime}$ as a function of the laser field detuning $\Delta_{l}$ and the ratio $\Omega_{p} / \Omega_{d}$ for $\gamma_{l w d}=500 \mathrm{MHz}, \gamma_{l w p}=2.5 \mathrm{GHz}$, high $\Omega_{p}$, and $\Omega_{d}=20 \gamma_{a c}$ in arbitrary units on a logarithmic scale.

appears to be possible. The peak intensity saturates more slowly and is reached only after lengths greater than $40 \mathrm{~mm}$. It is also an order of magnitude larger than in Fig. 11(a). Although interesting, this behavior is unlikely to be useful in a real scheme since the three level and other assumptions are violated in situations with such large detunings.

\section{The effect of laser linewidths on the behavior with two strong fields}

We now turn to the case where both laser fields are strong. Although not readily achievable in unfocused experiments relevant to VUV generation at present, this regime should be accessible for atomic schemes, such as $\mathrm{Li}$, in which all the relevant wavelengths are in the visible or near IR range. In Fig. 12 the ratio $R^{\prime}$ is plotted including laser linewidths. This is analogous to the case displayed in Fig. 8 for which the laser

linewidths were omitted. Most importantly we see that the form of the plot of $R^{\prime}$ versus $\Delta_{l}$ and $\Omega_{p} / \Omega_{d}$ is still related to that displayed in Fig. 8. At the very lowest values of $\Omega_{p}$ a single broad peak in the ratio is seen as a function of laser detuning. This evolves into a three peaked structure for modest $\Omega_{p}$ 's, which is explicable in terms of the dressing of the Autler-Townes satellites, by the pump field $\Omega_{p}$, already seen in Figs. 4 and 6. For higher $\Omega_{p}$ 's the three peaks split apart and grow, especially that at zero detuning. This corresponds to the appearance of negative values (gain) in $\operatorname{Im}\left[\chi^{A}\right]$. The vanishing of $R^{\prime}$ at $\Omega_{p} / \Omega_{d}=1.0$, due to destructive interference in $\left|\chi^{F W}\right|$, is also still present. A subsequent rise in $R^{\prime}$ for $\Omega_{p} / \Omega_{d}>1.0$ mirrors that in Fig. 8 .

These results clearly show strong evidence that the dressing of the atom by the strong fields still leads to extensive modifications to the nonlinear optical properties even when the laser linewidth is large. However, we cannot ignore the fact that for conditions with finite $\Omega_{p}$ there is gain present in this system (see Fig. 4). This gain also survives the inclusion of finite laser bandwidth. Since gain is present $R^{\prime}$ does not give a reliable guide to the total intensity generated, even if the phase matching is perfect. Therefore it is necessary to calculate the intensity using Eq. (34) which accounts not only for phase matching over a finite length, but also for the gain. Notice in this equation that the light intensity generated due to the gain grows exponentially with distance, therefore the relative importance of four-wave mixing and amplification through the medium is crucial. The four-wave mixing dominates at first, but as propagation distance increases the amplification effect becomes increasingly important. In effect the coherent light from four-wave mixing acts as a seed in the subsequent amplification. We plot results for a $1 \mathrm{mbar}$ gas density and a path length of $10 \mathrm{~mm}$ : under these conditions the generated intensity departs significantly from the form predicted by $R^{\prime}$ in Fig. 12. It should be noted that for a much shorter path length (e.g., $1 \mathrm{~mm}) R^{\prime}$ would be a good guide to the dependence in the generated intensity.

The most significant features in Fig. 13(a) are the three peaks at $\Delta=0$ and $\Delta= \pm 20 \gamma_{a c}$. The central peak which persists to higher values in $\Omega_{p} / \Omega_{d}$ arises from the near vanishing of absorption at zero detuning that evolves into a broad gain feature at higher pump fields (see Fig. 6). The feature here is due to both FWM and gain. The two satellite features are more localized in $\Omega_{p} / \Omega_{d}$ and $\Delta_{l}$ space and correspond to the strong gain features, associated with the Autler-Townes peaks when $\Omega_{p} / \Omega_{d}=0.1$, already seen in Figs. 4 and 6 . These are very pronounced features arising predominantly from the gain present at this frequency, and our ability to accurately calculate their magnitude is limited due to the uncertainty in the absolute magnitude of $\chi^{F W}$, and because a small $\Omega_{l}$ is an inherent assumption of our model. In order to evaluate the high intensity at the sidebands we have plotted in Fig. 13(b) this value as a function of the pump Rabi frequency $\Omega_{p}$.

\section{DISCUSSION}

Of the assumptions that we have made in modeling our system, the steady-state assumption is probably the most limiting. The requirement of intense laser fields often restricts us to the use of pulsed lasers (in most cases with typical pulse lengths of a few nanoseconds). This means that in order for the steady-state approximation to be valid we would require our frequencies (incoherent decays and Rabi frequencies) to be considerably larger than the $\sim 250 \mathrm{MHz}$ bandwidth of the laser. This figure is in fact close to the one we would expect for the radiative decays and the Rabi frequency of the pump transition in many VUV experiments so this will limit the utility of the approach in modeling pulsed experiments. Another outcome of requiring time independence for the matrix $M$ is that we cannot have more than one coherent coupling between any two levels. This removes any opportunity for including an electric quadrupole or magnetic dipole transition between $|a\rangle$ and $|c\rangle$.

The assumption of a plane-wave geometry is in general acceptable in pulsed experiments since (i) in the likely experiments this will be close to the actual situation since unfocused or weakly focused beams are likely to be used, and (ii) some qualitative features found in this limit would be expected to survive even if a tight focusing geometry is used. A treatment of focused Gaussian beams in a tight focusing limit, although more realistic for a $\mathrm{CW}$ experiment in which 
(a)
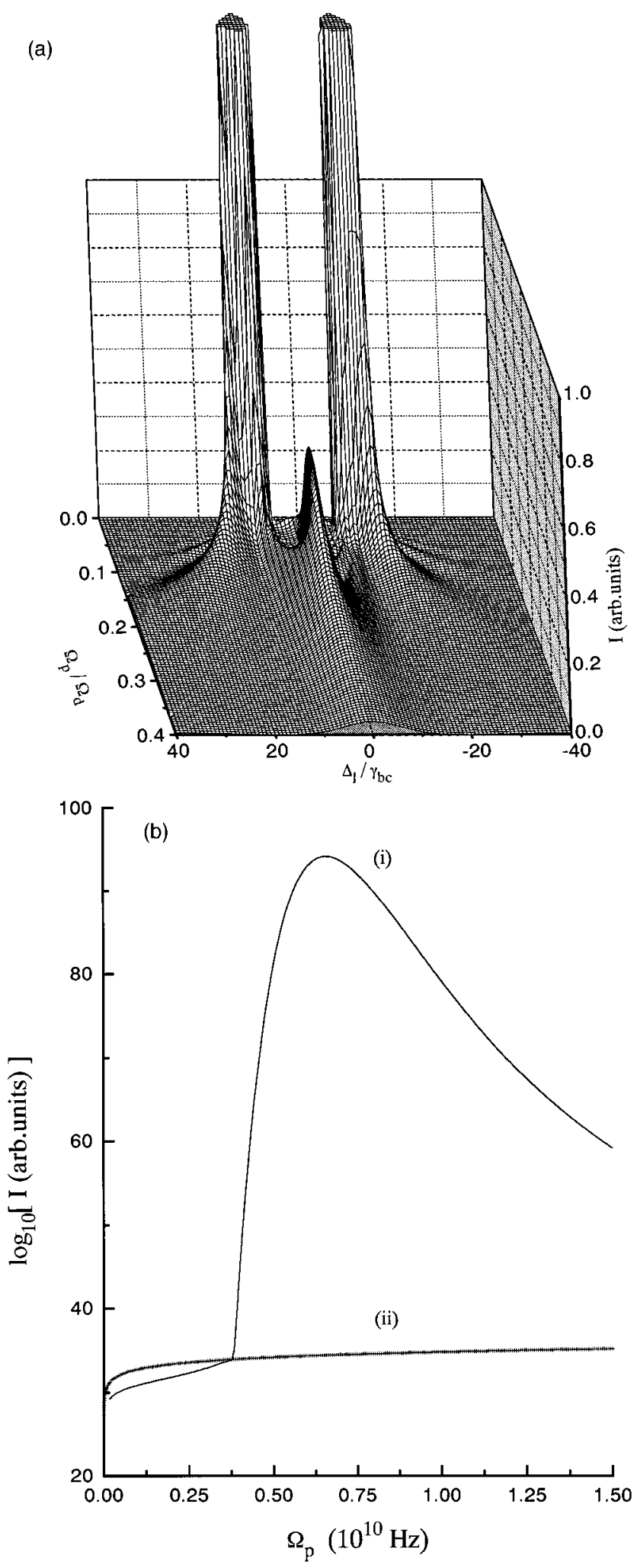

FIG. 13. The observable intensity $I$ as a function of the detuning $\Delta_{l}$ and the ratio $\Omega_{p} / \Omega_{d}$ (a) and two cross sections on a logarithmic scale (with basis 10) at $\Delta_{l}=0.5 \Omega_{l}[\mathrm{~b}(\mathrm{i})]$ and $\Delta_{l}=0[\mathrm{~b}$ (ii)] (at the distance $z=1 \mathrm{~cm}$ and pressure $p=1 \mathrm{mbar}$, laser linewidths $\gamma_{l w d}=500 \mathrm{MHz}, \gamma_{l w p}=2.5 \mathrm{GHz}$, and $\left.\Omega_{d}=20 \gamma_{a c}\right)$.

focusing is needed to achieve viable Rabi couplings, would in practice be more difficult to carry out. This is because of the additional complexity introduced by the intensity depen- dence of the susceptibilities, which would require calculation of the local single-atom response at each point in the focal volume. Moreover recent experiments have indicated the potential difficulties that will arise due to spatial modification of the laser beam arising from the intensity dependence of $\operatorname{Re}\left[\chi^{A}\right][13]$.

We have not included any competing processes such as photoionization and multiphoton ionization which can be important under certain circumstances. Although the atomic schemes were chosen such that direct single photon ionization out of excited states $|a\rangle$ and $|b\rangle$ is not possible from the field at $\omega_{d}$, single photon ionization from state $|a\rangle$ due to the field at $\omega_{p}$ can occur. Moreover the large value of $\Omega_{d}$ makes multiphoton ionization rates appreciable. These are not included in our calculation even though they could have two effects. The first of these is the direct effect on the atomic response caused by depletion of population (the three-level system is no longer closed) and any additional accompanying lifetime broadening. Second, when the photoelectron density becomes significant the phase matching due to contributions from these electrons will need to be included.

An important feature of our work is that in the results presented we are trying to use experimentally realistic parameters. We use real atomic transition strengths and wavelengths together with realistic laser bandwidths and Rabi frequencies for pulsed laser systems operating at these wavelengths enabling us to use the theory to provide realistic predictions for, e.g., $\chi^{A}$ and hence the absorption length and the achievable degree of dispersion. We also take advantage of algebraic computations to invert the fully dimensional matrix governing the dynamics of the density operator which allows us to stay away from the more restrictive assumptions in many of the models in the literature. These include assuming the populations of the excited states to be zero and only treating $\Omega_{p}$ to first order. By keeping $\Omega_{d}$ and $\Omega_{p}$ to all orders we can investigate a larger range of parameters and are not restricted to $\Omega_{d} \gg \Omega_{p}$. This allows us to look at situations where $\Omega_{d} \approx \Omega_{p}$.

We are also exploring a regime in which "gain" due to the negative value of $\operatorname{Im}\left[\chi^{A}\right]$ at $\omega_{l}$ is present simultaneously with a source of field at $\omega_{l}$ due to $\left|\chi^{F W}\right|$. This is an unusual situation and has several interesting consequences. As was explained above, the relative importance of the two processes depends on the path length. The growth of the field due to gain depends exponentially on the path length, while that due to FWM depends in detail on the phase matching. Over short distances the build up of the field due to stimulated emission will be small and here we would expect radiation generated by four-wave mixing to dominate. The two processes will, however, be coupled, with the four-wave mixing, in effect, acting as a seed for the amplification by stimulated emission. This then means that, providing the competition from amplified spontaneous emission is small, we have a radiation source with coherence and other properties controlled by those of the input fields, as in a normal parametric process, but with very significant additional amplification due to the gain. We believe that this situation, unique for strong field resonant FWM, could lead to a potentially exploitable VUV light source. 


\section{CONCLUSIONS}

We have explored the efficiency of four-wave mixing under the influence of electromagnetically induced transparency on two nonlinearly driven transitions. We have derived a variable $R^{\prime}$ governing this efficiency differing for nonvanishing detunings $\Delta_{l}$ from previously known results and find it drastically enhanced when both Rabi frequencies $\Omega_{p}$ and $\Omega_{d}$ are large and comparable. We have estimated a realistic range of parameters using data of various noble gases and estimated the extent under which the effect of enhanced fourwave mixing is reduced due to the influence of Doppler broadening, laser phase fluctuations, and phase mismatching. A resonant enhancement in four-wave mixing is still observable under the realistic conditions considered here.

When both fields are strong we see, in addition to fourwave mixing, that strong gain can occur at certain frequencies. This arises due to Raman pumping of population into the upper levels; the vanishing absorption then leads to a gain without inversion. In the presence of seed light from the accompanying four-wave-mixing process we predict that amplification will occur in this situation.

\section{ACKNOWLEDGMENTS}

This work was supported in part by the UK Engineering and Physical Sciences Research Council and by the European Community. The authors would like to thank Carsten Szymanowski for helpful discussions.

\section{APPENDIX A}

The purpose of this appendix is to show that Eq. (35) can be derived in a straightforward manner from Eq. (33), which can also be written in the form

$$
\frac{\partial}{\partial z} A_{l}+P A_{l}=Q e^{-i \Delta k_{l} z}
$$

where

$$
\begin{gathered}
P=\frac{\omega}{2 c}\left\{\operatorname{Im}\left[\chi^{A}\right]-i \operatorname{Re}\left[\chi^{A}\right]\right\} \\
Q=i \frac{\omega}{4 c} \chi^{F W}\left(-\omega_{l}, 2 \omega_{p},-\omega_{d}\right) A_{p}^{2} A_{d}^{*}
\end{gathered}
$$

for $\omega=\omega_{l}$. We would like to emphasize that these susceptibilities include the number density $N$ as in Eq. (25). This is a linear first-order differential equation with nonzero RHS that can be solved easily, for example following the method described in [28]. The solution is given by

$$
A_{l}=e^{-I} \int Q e^{-i \Delta k_{l} z} e^{I} d z+c e^{-I}
$$

where $I=\int P d z$ so, in our case,

$$
\begin{gathered}
I=P z, \\
A_{l}=e^{-P z} Q \int e^{\left(P-i \Delta k_{l}\right) z} d z+c e^{-P z} \\
=Q e^{-P z}\left[1 /(P-i \Delta k) e^{(P-i \Delta k) z}\right]_{0}^{z}+c e^{-P z} \\
=Q e^{-P z}\left[\left(e^{(P-i \Delta k) z}-1\right) /(P-i \Delta k)\right]+c e^{-P z} .
\end{gathered}
$$

The boundary condition of $A_{l}(z=0)=0$ can now be applied:

$$
0=c
$$

leading to

$$
A_{l}=\frac{Q}{(P-i \Delta k)}\left[e^{-i \Delta k z}-e^{-P z}\right]
$$

and

$$
A_{l}^{*}=\frac{Q^{*}}{\left(P^{*}+i \Delta k\right)}\left[e^{+i \Delta k z}-e^{-P^{*} z}\right]
$$

We can now go from this to the intensity via the relation

$$
I=\frac{2 n}{Z_{0}}|A|^{2}
$$

(see [29]).

$$
I=\frac{2 n}{Z_{0}}|Q|^{2} \frac{\left[1+e^{-\left(P+P^{*}\right) z}-e^{-i \Delta k z} e^{-P^{*} z}-e^{+i \Delta k z} e^{-P z}\right]}{|P|^{2}+\Delta k^{2}+i \Delta k\left(P-P^{*}\right)}
$$

now, from our definition of $P$ and $Q$,

$$
\begin{gathered}
P-P^{*}=-i \frac{\omega}{c} \operatorname{Re}\left[\chi^{A}\right] \\
|P|^{2}=\frac{\omega^{2}}{4 c^{2}}\left|\chi^{A}\right|^{2} \\
P+P^{*}=\frac{\omega}{c} \operatorname{Im}\left[\chi^{A}\right], \\
|Q|^{2}=\frac{9 \omega^{2}}{16 c^{2}}\left|\chi^{F W}\right|^{2}\left|A_{p}\right|^{4}\left|A_{d}\right|^{2},
\end{gathered}
$$

thus

$$
\begin{aligned}
& I=\frac{2 n}{Z_{0}}|Q|^{2} \frac{\left[1+e^{-(\omega / c) \operatorname{Im}\left[\chi^{A}\right] z}-e^{-(\omega / 2 c) \operatorname{Im}\left[\chi^{A}\right] z}\left(e^{\left\{-i \Delta k-(\omega / 2 c) \operatorname{Re}\left[\chi^{A}\right]\right\} z}+\text { c.c. }\right)\right]}{\left(\omega^{2} / 4 c^{2}\right)\left|\chi^{A}\right|^{2}+\Delta k^{2}+(\omega / c) \Delta k \operatorname{Re}\left[\chi^{A}\right]}, \\
& I=\frac{2 n}{Z_{0}}|Q|^{2} \frac{\left[1+e^{-(\omega / c) \operatorname{Im}\left[\chi^{A}\right] z}-2 e^{-(\omega / 2 c) \operatorname{Im}\left[\chi^{A}\right] z} \cos \left(\left\{\Delta k+(\omega / 2 c) \operatorname{Re}\left[\chi^{A}\right]\right\} z\right)\right]}{\left(\omega^{2} / 4 c^{2}\right) \operatorname{Im}\left[\chi^{A}\right]^{2}+\left\{\Delta k+(\omega / 2) c \operatorname{Re}\left[\chi^{A}\right]\right\}^{2}}
\end{aligned}
$$


which leads us finally to Eq. (35).

\section{APPENDIX B}

We devote this appendix to the estimation of the main variables in our model. These are $\Omega_{d}, \Omega_{p}$, and the detunings, which we will treat in this order. As the transition between $|a\rangle$ and $|b\rangle$ is an E1 allowed transition, we can use the simple relation given in Eq. (5):

$$
\hbar \Omega_{d}=\mu_{a b}\left|E\left(\omega_{d}\right)\right| .
$$

The dipole moment matrix element $\mu_{a b}$ can be rewritten in terms of the Einstein $A$ coefficient:

$$
A_{a b}=\frac{\omega_{a b}^{3}}{3 \pi \epsilon_{0} \hbar c^{3}} \sum_{m_{j}}|\langle a|e \cdot r| b\rangle|^{2}
$$

(see [30]). It is of course important to consider the degeneracies. In the case of two nondegenerate levels, the dipole moment refers to the line strength. If the calculation of the Rabi frequencies is performed including degeneracy then they will depend on a three- $j$ symbol (see [16]). This means that when we consider a degenerate two level system there is no single Rabi frequency. The states $|a\rangle$ and $|c\rangle$ in $\mathrm{Kr}$ are $J=0$, and the $|b\rangle$ state is $J=1$. If linearly polarized light is used the allowed transitions within the $g_{j}$ degenerate sublevels are limited to one, leading to population trapping in the $|b\rangle m_{j= \pm 1}$ states due to the spontaneous decays. This will be a small factor effecting the population and can be ignored. The available data on the $\mathrm{Kr}$ transitions is in the form of Einstein A coefficients (or lifetimes). Since the spontaneous decay occurs in all polarizations, this refers to the decay from $|a\rangle_{J=0, m_{j}=0}$ to any of the $|b\rangle_{J=1, m_{j}=0, \pm 1}$ states. What we are specifically interested in is the transition rate between $|a\rangle_{m_{j}=0}$ and $|b\rangle_{m_{j}=0}$. The branching ratios of the so-called $J_{c} l$ coupled system can be approximated by that of a RusselSaunders coupling. This gives the $p$ ratio to be twice that of the $s$ 's so a factor of a half is phenomenologically introduced:

$$
\Omega_{d}=\left(\frac{1}{2} A_{a b} \times \frac{3 \pi \epsilon_{0} c^{3}}{\hbar \omega_{d}^{3}}\right)^{\frac{1}{2}} \times\left|E\left(\omega_{d}\right)\right|
$$

given we know $A_{a b}$ it only remains to estimate the $\left|E\left(\omega_{i j}\right)\right|$.

The relationship between intensity and the modulus of the electric field for plane polarized, collimated light is

$$
I=\frac{1}{2} n c \epsilon_{0}\left|E\left(\omega_{i j}\right)\right|^{2}
$$

(see [30]) and between the energy of the pulse and the intensity:

$$
I=\frac{E}{t A},
$$

where $E$ designates the energy of the pulse in Joules, $I$ the pulse duration in seconds, and $A$ the cross-sectional area in square meters. Then finally

$$
\left|E\left(\omega_{i j}\right)\right|=\left(\frac{2 E}{n c \epsilon_{0} \tau A}\right)^{\frac{1}{2}}
$$

which leads to

$$
\Omega_{d}=\left(\frac{1}{2} A_{a b} \times \frac{3 \pi \epsilon_{0} c^{3}}{\hbar \omega_{d}^{3}}\right)^{\frac{1}{2}} \times\left(\frac{2 E}{n c \epsilon_{0} \tau A}\right)^{\frac{1}{2}} .
$$

The laser system used in our experiments delivers $50 \mathrm{~mJ}$ in a $4 \mathrm{~ns}$ pulse with a beam diameter of approximately $5 \mathrm{~mm}$. This gives rise to an unfocused electric field strength of

$$
\left|E_{\text {unfocused }}\right| \approx 21.9 \times 10^{6} \mathrm{Vm}^{-1} .
$$

For the $\omega_{a b}$ transition, the $g_{a}=1, A_{a b}=49 \times 10^{6} \mathrm{~s}^{-1}$ (see [17]), and $\omega_{b c}=95 \times 10^{16} \mathrm{rad} \mathrm{s}^{-1}$. This gives us

$$
\Omega_{d M A X} \approx 1.6 \times 10^{6} \times\left|E_{d}\right| \approx 3.5 \times 10^{13} \mathrm{rad} \mathrm{s}^{-1} .
$$

Obviously this is only an estimation, but it tells us that $\Omega_{d}$ 's of the order of a few $\mathrm{THz}$ should be reachable.

The estimation of $\Omega_{p}$ is less exact due to the lack of any experimental data on the transition strength of the twophoton transition. For a two photon transition we have

$$
\hbar \Omega_{i j}=\frac{1}{2} \sum_{q, \text { allstates }} \frac{\left(d_{1 q} E_{p}^{*}\right)\left(d_{q 2} E_{p}^{*}\right)}{E_{1}-E_{q}+\frac{1}{2} \hbar \omega_{i j}}\left|E_{p}\right|^{2}
$$

(see [16]). Looking at the energy level structure of $\mathrm{Kr}$ we can see the additional levels all lie above the $|a\rangle$ state in Fig. 1. This implies that most of the two-photon coupling strength comes from the level $|b\rangle$. So, if the only level we sum over is $|b\rangle$ then we can simplify (A7) to

$$
\hbar \Omega_{p} \approx \frac{\frac{1}{2} \mu_{b c} \mu_{a b}}{\hbar\left(\omega_{b c}-\frac{1}{2} \omega_{a c}\right)}\left|E_{p}\right|^{2} .
$$

Here again the $\mu_{i j}$ 's refer to the line strengths rather than the dipole moments. The calculation of $\mu_{a b}$ is done as in the treatment for $\Omega_{d}$, i.e., with the factor of $\frac{1}{2}$. For $\mu_{b c}$ we also have a $J=1 \rightarrow J=0$ transition. The experimental data are given in terms of absorption oscillator strengths; $f_{c b}=0.155 \pm 0.011$. Again this is absorption from $|c\rangle_{J=0, m_{j}=0} \rightarrow|b\rangle_{J=1, m_{j}=0, \pm 1}$ and we want the transition strength between $|c\rangle_{J=0, m_{j}=0} \rightarrow|b\rangle_{J=1, m_{j}=0}$. The transfer from $f_{c b}$ to $A_{b c}$ is given by

$$
A_{b c}=\frac{g_{c}}{g_{b}} \frac{e^{2} \omega_{l}^{2}}{2 \pi \epsilon_{0} m c^{3}} f .
$$

This leads to

$$
\begin{aligned}
\mu_{b c} & \approx 6.98 \times 10^{-30} \mathrm{Cm}, \\
\mu_{a b} & \approx 1.21 \times 10^{-30} \mathrm{Cm},
\end{aligned}
$$

and thus to 


$$
\Omega_{p} \approx 5.95 \times 10^{-8}\left|E_{p}\right|^{2} .
$$

The pump laser field is provided by an excimer pumped dye laser that produces a $20 \mathrm{~ns}$ pulse of $212 \mathrm{~nm}$ radiation with an energy of $0.5 \mathrm{~mJ}$. The beam diameter is also approximately $5 \mathrm{~mm}$ giving rise to an unfocused electric field strength of $\left|E_{p}\right|=9.79 \times 10^{5}$. So, all together we get an order of magnitude estimate of $\Omega_{p M A X} \sim 6 \times 10^{4} \mathrm{rad} \mathrm{s}^{-1}$ for the unfocused case.

Obviously there is no experimental constraint on the detunings (but the theory will break down if $\Delta / \Omega \gg 1$ ) but there is a choice to be made on the detuning scheme. From energy conservation we have

$$
\Delta_{p}=\Delta_{d}+\Delta_{l}
$$

If we tune the driving laser while keeping the pump field on resonance, then we have $\Delta_{p}=0$, and $\Delta_{d}=\Delta_{l}$. Because the effect we are interested in is electromagnetically induced transparency, the most advantageous case is for the driving field to be on resonance at all times. So for the results presented in this paper we have

$$
\begin{aligned}
& \Delta_{d}=0, \\
& \Delta_{p}=\Delta_{l},
\end{aligned}
$$

and most of the line shapes will be given as a function of $\Delta$.
[1] G. Alzetta et al., Nuovo Cimento B36, 5 (1976); R.M. Whitley and C.R. Stroud, Jr., Phys. Rev. A 14, 1498 (1976); P. M. Radmore and P. L. Knight, J. Phys. B 15, 561 (1982); K.-J. Boller, A. Imamoglu, and S. E. Harris, Phys. Rev. Lett. 66, 2593 (1991); J. E. Field et al., ibid. 67, 3062 (1991).

[2] O. Kocharovskaya and Ya. I. Khanin, Pis'ma Zh. Éksp. Teor. Fiz. 90, 1610 (1986) and 48, 581 (1988) [JETP Lett. 48, 630 (1988)]; S. E. Harris, Phys. Rev. Lett. 62, 1033 (1989); M. O. Scully, S.-Y. Zhu, and A. Gavriliedes, ibid. 62, 2813 (1989); A. Lyras et al., Phys. Rev. A 40, 4131 (1989).

[3] S.E. Harris and J. J. Macklin, Phys. Rev. A 40, 4135 (1989); A. Imamoglu, ibid. 40, 2835 (1989); S. Basile and P. Lambropoulos, Opt. Commun. 78, 163 (1990); G. S. Agarwal, S. Ravi, and J. Cooper, Phys. Rev. A 41, 4721 (1990); 41, 4727 (1990); O. Kocharovskaya and P. Mandel, ibid. 42, 523 (1990); E. E. Fill, M. O. Scully, and S. Y. Zhu et al., Opt. Commun. 77, 36 (1990); L. M. Narducci et al., ibid. 81, 379 (1991); 86, 324 (1991); C. H. Keitel et al., Phys. Rev. A 48, 3196 (1993).

[4] J. Gao et al., Opt. Commun. 93, 323 (1992); A. Nottelmann, C. Peters, and W. Lange, Phys. Rev. Lett. 70, 1783 (1993); E. Fry et al., Phys. Rev. Lett. 70, 3235 (1993); W. E. van der Veer et al., ibid. 70, 3243 (1993).

[5] M. O. Scully, Phys. Rev. Lett. 67, 1855 (1991); M. Fleischhauer et al., Phys. Rev. A 46, 1468 (1992); A. D. WilsonGordon and H. Friedmann, Opt. Commun. 94, 238 (1992); U. Rathe et al., Phys. Rev. A 47, 4994 (1993); C. Szymanowski and C. H. Keitel, J. Phys. B 27, 5795 (1994), C. Szymanowski et al., J. Mod. Opt. 42, 985 (1995).

[6] H. Ritsch, M.A.M. Marte, and P. Zoller, Europhys. Lett. 19, 7 (1992); K. M. Gheri and D. F. Walls, Phys. Rev. A 45, 6675 (1992); A. S. Manka et al., Opt. Commun. 94, 174 (1992).

[7] O. Kocharovskaya, Phys. Rep. 219, 175 (1992); M. O. Scully, ibid. 191 (1992); P. Mandel, Contemp. Phys. 34, 235 (1993).

[8] S.E. Harris, J.E. Field, and A. Imamoglu, Phys. Rev. Lett. 64, 1107 (1990).
[9] K. Hakuta, L. Marmet, and B.P. Stoicheff, Phys. Rev. Lett. 66, 596 (1991).

[10] G.Z. Zhang, K. Hakuta, and B.P. Stoicheff, Phys. Rev. Lett. 71, 3009 (1993).

[11] M. Jain et al., Opt. Lett. 18, 998 (1993).

[12] P.J. Harsham et al., Opt. Lett. 18, 1706 (1993).

[13] M. Xiao et al., Phys. Rev. Lett. 74, 666 (1995); R.R. Moseley et al., ibid. 670 (1995).

[14] H. Haken, Handbuch der Physik, edited by S. Flugge (Springer, Berlin, 1970), Vol. 25.

[15] S. Wolfram, Mathematica, 2nd ed. (Addison-Wesley, Redwood City, 1991).

[16] B.W. Shore, The Theory Of Coherent Atomic Excitation (John Wiley and Sons, New York, 1990).

[17] W. E. Ernst and E. Schulz-Gulde, Physica 93C, 136 (1978).

[18] L.M. Narducci et al., Phys. Rev. A 42, 1630 (1990); A.S. Manka et al., ibid. 3748 (1991).

[19] J.F. Reintjes, Nonlinear Optical Parametric Processes in Liquids and Gases (Academic Press, Boston, 1984).

[20] K. Ness, Ph. D. thesis, Imperial College, London, 1984 (unpublished).

[21] R.J. Glauber, in Quantum Optics and Electronics, edited by C. de Witt, A. Blandin, and C. Cohen-Tannoudji (Gordon and Breach, New York, 1965).

[22] P.A. Lakshmi and S. Swain, J. Mod. Opt. 38, 2031 (1991); S. Sultana and M.S. Zubairy, Phys. Rev. A 49, 438 (1994).

[23] G.S. Agarwal, Phys. Rev. A 18, 1490 (1978).

[24] K.I. Osman and S. Swain, J. Phys. B 13, 2397 (1980).

[25] B.J. Dalton and P.L. Knight, J. Phys. B 15, 3997 (1982).

[26] G. Hilber, A. Lago, and R. Wallenstein, J. Opt. Soc. Am. B 4, 1753 (1987).

[27] J.P. Marangos et al., J. Opt. Soc. Am. B 7, 1254 (1990).

[28] M.L. Boas, Mathematical Methods in the Physical Sciences (John Wiley and Sons, New York, 1966).

[29] R.W. Boyd, Nonlinear Optics (Academic Press, Boston, 1992).

[30] A.P. Thorne, Spectrophysics, 2nd ed. (Chapman and Hall, New York, 1988). 